\title{
Un Modelo de Credit Scoring para instituciones de microfinanzas en el marco de Basilea II
}

\section{A Credit Scoring Model for Institutions of Microfinance under the Basel II Normative}

\author{
Salvador Rayo Cantón ${ }^{1}$ \\ Juan Lara Rubio ${ }^{2}$ \\ David Camino Blasco ${ }^{3}$
}

\begin{abstract}
Resumen
El crecimiento de los microcréditos a nivel mundial, junto con la normativa internacional sobre requerimientos de capital (Basilea II), están impulsando a las instituciones de microfinanzas (IMFs) a una mayor competencia con las entidades bancarias por este segmento de negocio. La banca tradicionalmente ha contado con adecuados modelos de credit scoring para analizar el riesgo de incumplimiento, pero esto no ha sido así en las IMFs supervisadas. El objetivo de esta investigación es diseñar un modelo de credit scoring para una institución sometida a supervisión y especializada en microcréditos, como es la Entidad de Desarrollo de la Pequeña y Micro Empresa (Edpyme) del sistema financiero del Perú. El resultado de la investigación muestra la metodología y fases necesarias para diseñar el modelo, así como el proceso de valoración y validación para que pueda ser aplicado en el área de negocio, especialmente para establecer la política de tasas de interés con clientes. Por último, también se muestra cómo puede utilizarse el modelo para desarrollar una gestión del riesgo de crédito en el marco de los métodos IRB de Basilea II.
\end{abstract}

Palabras clave: Microcréditos, instituciones de microfinanzas, Basilea II, credit scoring, Logit, IRB

\begin{abstract}
The growth of microcredit worldwide along with international rules on capital requirements (Basel II) are increasing the competition between microfinance institutions (MFIs) and banks for this business segment. The bank system traditionally has relied on adequate credit scoring models to analyze the risk of payment failures, but this has not been the case in supervised MFIs. The objective of this research is to design a credit scoring model for any institution subjected to supervision and specialized in microcredit as the Development Agency for Small and Micro Enterprise (Entidad de Desarrollo de la Pequeña y Micro Empresa - Edpyme) of the financial system in Peru. The results of this research includes a methodology and the steps needed to design the model, and the assessment and validation process that can be applied in the business area, in particular, to establish an interest rate policy with customers. Eventually, the paper also explains how the model can be used to develop credit risk management under the Basel II IRB approaches.
\end{abstract}

Keywords: Microcredit, institutions of microfinance, Basel II, credit scoring, Logit, IRB

1. Profesor del Departamento de Economía Financiera y Contabilidad, Universidad de Granada, España; $<$ srayo@ugr.es $>$

2. Profesor del Departamento de Economía Financiera y Contabilidad, Universidad de Granada, España; <juanlara@ugr.es $>$

3. Profesor del Departamento de Economía de la Empresa, Universidad Carlos III de Madrid, España; <dcamino@emp.uc3m.es> 


\section{INTRODUCCIÓN}

El acuerdo internacional sobre regulación y supervisión bancaria denominado "Nuevo Acuerdo de Capital", aprobado en 2004 por el Comité de Basilea y conocido como Basilea II, exige a las entidades financieras de los países que se adscriba al mismo una revisión de sus dotaciones de capital para cubrir los riesgos. Por consiguiente, las entidades financieras y de microfinanzas (IMFs) supervisadas requieren adoptar procesos internos que sean capaces de medir el riesgo de crédito. Las entidades financieras que sirven a la industria microfinanciera deberán actuar bajo los parámetros de Basilea II. En efecto, estos parámetros les obligan a disponer de herramientas que les permitan establecer modelos de medición (scorings y ratings) con objeto de discriminar a los clientes según su perfil de riesgo, sistemas de seguimiento del riesgo vivo y modelos de evaluación de la exposición y la severidad en el riesgo de crédito. Basilea II también obliga a las entidades financieras no solo a adaptar sus sistemas de cálculo del consumo de capital, sino también a modificar los sistemas de reporting (informes financieros) y de análisis de la información. Ambos elementos, es decir los informes financieros y el análisis de los mismos, son la clave de Basilea II que hace necesario gestionar grandes bases de datos, capaces de ofrecer la información exacta para cuantificar los riesgos de cada operación, lo que supone un verdadero reto para los bancos y, especialmente, para las entidades de microfinanzas.

La explicación y predicción del riesgo de impago en microfinanzas deben ser abordadas de una manera distinta a la habitual en la banca comercial debido a las limitaciones de las bases de datos y al proceso de elaboración de un sistema de medición del riesgo de microcrédito. Preguntas tales como: ¿conviene este cliente?, ¿cuál es el límite de crédito que debo aceptarle en su solicitud?, ¿qué tasa de interés debo cobrarle?, ¿cómo puedo reducir el riesgo de impago?, etc., son cuestiones que deben acompañarse de una respuesta acertada, dado un determinado entorno macroeconómico. Historiales de crédito poco desarrollados y faltos de información impiden predecir la probabilidad del no pago de deudas mediante el uso de los sistemas tradicionales de credit scoring desarrollados para la banca. En este sentido, la incertidumbre provocada por el entorno macroeconómico sugiere la importancia de un sistema efectivo de control de riesgos. En consecuencia, planteamos un método que pueda resolver el problema de una correcta clasificación de clientes, ya sean cumplidos o morosos. Para ello, diseñamos una metodología que analizará el riesgo de impago en la concesión de microcréditos.

En el Perú, el Reglamento para el Requerimiento de Patrimonio Efectivo por Riesgo de Crédito de la Superintendencia de Banca y Seguros (SBS) del 2009 constituye un notable avance para la implementación de Basilea II entre las entidades sometidas a supervisión, entre ellas las Entidades de Desarrollo de la Pequeña y Micro Empresa (Edpyme). Si bien la norma establece un modelo estándar para la gestión del riesgo de crédito, también ofrece la posibilidad de que las IMFs puedan implementar modelos internos. Es decir, la nueva normativa derivada de este reglamento aporta como principal novedad la posibilidad de desarrollar Métodos Basados en Calificaciones Internas (IRB) que deberán estar homologados por la Superintendencia de Banca y Seguros. Por tanto, es necesario utilizar métodos estadísticos más precisos para la estimación de la probabilidad de insolvencia y de la severidad. Ambas estimaciones son fundamentales para calcular el requerimiento de capital, la pérdida esperada y la pérdida inesperada, aspectos claves para establecer la política de tasas de interés. En este nuevo contexto se requiere contar no solo con nuevos sistemas y metodologías estadísticas y econométricas, sino también, con profesionales con la formación adecuada para desarrollar e implantar en la institución financiera los modelos adecuados de estimación de la insolvencia y la severidad. En definitiva, Basilea II constituye un nuevo reto para las entidades de microfinanzas y para los profesionales que laboran en ellas.

Desde nuestra perspectiva, el elemento clave para analizar el proceso de calificación de riesgos mediante modelos internos (IRB), según la normativa de Basilea II, es que las IMFs dispongan de un modelo de 
credit scoring que les permita medir la probabilidad de impago del crédito que se otorga. Para tal efecto, el método estándar posibilitaría la formulación de los métodos IRB. Este proceso se analiza en el presente trabajo estructurado en seis partes. Luego de la Introducción, la segunda parte plantea un marco teórico donde se exponen los principales modelos de credit scoring que se utilizan en las entidades bancarias para el análisis del riesgo de impago. En ese mismo apartado recopilamos las aplicaciones más relevantes en cuanto a riesgo de impago de las IMFs. La tercera parte del trabajo describe la metodología empleada en la consecución de un modelo de credit scoring para instituciones de microfinanzas. Hemos utilizado como base de investigación la cartera de créditos para microempresas de la Edpyme Proempresa, entidad del sistema financiero del Perú. En el cuarto epígrafe presentamos el desarrollo metodológico y los resultados de la aplicación del credit scoring para la Edpyme Proempresa. En el quinto apartado aplicamos el modelo de credit scoring obtenido en las decisiones de negocio y en la política de pricing mediante un modelo IRB avanzado en el marco de Basilea II. En sexto y último lugar proponemos una serie de conclusiones derivadas de la investigación.

\section{MARCO TEÓRICO}

\section{Concepto de Credit Scoring}

Los credit scoring, según Hand and Henley (1997), son procedimientos estadísticos que se usan para clasificar a aquellos que solicitan crédito, inclusive a los que ya son clientes de la entidad crediticia, en los tipos de riesgo 'bueno' y 'malo'. En sus inicios, durante los años setenta, las aplicaciones del credit scoring se construían con técnicas estadísticas (en particular, con el análisis discriminante). Posteriormente, los métodos empleados evolucionaron a técnicas matemáticas, econométricas y de inteligencia artificial. En cualquier caso, la construcción de toda aplicación del credit scoring se realiza tomando la información del cliente contenida en las solicitudes del crédito, de fuentes internas e, incluso, de fuentes externas de información.
El credit scoring estima el momento en el que se está llevando a cabo la solicitud, cuál será el comportamiento del crédito hasta su vencimiento, atendiendo al riesgo del cliente. Se evalúa a través de un modelo predictivo de comportamiento de pago o reembolso mediante una puntuación que mide el riesgo de un prestatario y/o de la operación. En general, estos métodos de calificación de créditos se aplican para obtener un conocimiento sobre distintos aspectos tales como los siguientes:

a) el comportamiento financiero en cuanto a los productos solicitados y a la morosidad;

b) la relación entre el riesgo y rentabilidad. El credit scoring aporta información sobre el precio o prima por riesgo, volatilidad, diversificación, etc.;

c) el coste de la operación. La agilización general de procesos que se consigue con el credit scoring permite la reducción del coste en el proceso de concesión de un crédito.

\section{Modelos de Credit Scoring en la banca}

A continuación presentamos un resumen de los principales trabajos en materia de credit scoring en la banca, agrupados según la metodología estadística aplicada.

\section{a) Análisis Discriminante}

El análisis discriminante consiste en una técnica multivariante que permite estudiar simultáneamente el comportamiento de un grupo de variables independientes con la intención de clasificar una serie de casos en grupos previamente definidos y excluyentes entre sí (Fisher, 1936). La principal ventaja de esta técnica está en la diferenciación de las características que definen cada grupo, así como las interacciones que existen entre ellas. Se trata de un modelo apropiado para clasificar buenos y malos pagadores a la hora de reembolsar un crédito. Entre los inconvenientes que presenta el análisis discriminante está la rigidez para cumplir las hipótesis de partida (linealidad, normalidad, homocedasticidad e independencia) y, sobre todo, la incapacidad para el cálculo de las probabilidades de impago.

Altman (1968) desarrolló la metodología más utilizada para pronosticar la insolvencia empresarial aplicando 
variables explicativas en forma de ratios. La Z-score de Altman se interpretaba a través de las variables ingresos netos/ventas, ganancias retenidas/activos, EBIT/activos, valor de mercado del patrimonio neto/valor libros de la deuda y ventas/activos. Esta metodología se adaptó posteriormente a la predicción de la morosidad de clientes de entidades bancarias.

\section{b) Modelos de Probabilidad Lineal}

Los modelos de probabilidad lineal utilizan un enfoque de regresión por cuadrados mínimos, donde la variable dependiente (variable dummy) toma el valor de uno (1) si un cliente es fallido, o el valor de cero (0) si el cliente cumple con su obligación de pago. La ecuación de regresión es una función lineal de las variables explicativas. Orgler (1970) fue el precursor de esta técnica usando el análisis de regresión en un modelo para préstamos comerciales. Este mismo autor recurrió a dicha técnica para construir un modelo de credit scoring para préstamos al consumo (Orgler, 1971), destacando el alto poder predictivo de las variables sobre el comportamiento del cliente, clasificadas fundamentalmente en cuatro grandes grupos: liquidez, rentabilidad, apalancamiento y actividad.

\section{c) Modelos Logit}

Los modelos de regresión logística permiten calcular la probabilidad que tiene un cliente para pertenecer a uno de los grupos establecidos a priori (no pagador o pagador). La clasificación se realiza de acuerdo con el comportamiento de una serie de variables independientes de cada observación o individuo. La principal ventaja del modelo de regresión logística radica en que no es necesario plantear hipótesis de partida, como por ejemplo la normalidad de la distribución de las variables, mejorando el tratamiento de las variables cualitativas o categóricas. Además, este modelo presenta la ventaja de medir la probabilidad de incumplimiento al mantener la variable explicada siempre dentro de un rango de variación entre cero y uno. Wiginton (1980) fue uno de los primeros autores en publicar un modelo de credit scoring aplicando esta metodología. Este autor realizó un estudio comparado entre el análisis discriminante y el modelo Logit en el que determinó que dicho modelo ofrecía un porcentaje de clasificación mejor que el análisis discriminante.

\section{d) Modelos de Programación Lineal}

Método encuadrado dentro de los modelos no paramétricos de credit scoring. En general, este tipo de modelos presentan mayor validez cuando se desconoce la forma que pueda mantener la relación funcional entre las variables. Los modelos de programación lineal permiten programar plantillas o sistemas de asignación de rating sin perder de vista el criterio de optimización de clientes correctamente clasificados. Hand (1981), Showers y Chakrin (1981) y Kolesar y Showers (1985) sentaron las bases de aplicabilidad de esta técnica en la actividad bancaria; a partir de ellos, otros autores han desarrollado esta metodología para predecir la omisión de pago de créditos.

\section{e) Redes Neuronales}

Es una metodología catalogada dentro de las técnicas no paramétricas de credit scoring. Las redes neuronales artificiales tratan de imitar al sistema nervioso, de modo que construyen sistemas con cierto grado de inteligencia. La red está formada por una serie de procesadores simples, denominados nodos, que se encuentran interconectados entre sí. Como nodos de entrada consideramos las características o variables de la operación de crédito. El nodo de salida sería la variable respuesta definida como la probabilidad de no pago. La finalidad de cada nodo consiste en dar respuesta a una determinada señal de entrada. El proceso de credit scoring mediante el uso de esta técnica resulta complicado, pues el proceso interno de aprendizaje funciona como una "caja negra" (capa oculta), donde la comprensión de lo que ocurre dentro requiere de conocimientos especializados.

Davis, Edelman y Gammerman (1992) publicaron un trabajo comparando esta técnica con otras técnicas alternativas de clasificación de clientes. Con posterioridad, Ripley (1994) y Rosenberg y Gleit (1994) describieron algunas de las aplicaciones de las redes neuronales 
empleadas en las decisiones gerenciales sobre el crédito y sobre la detección del fraude. Desde entonces, gracias al avance en nuevas tecnologías, se han diseñado sistemas avanzados para el objetivo de la clasificación de 'buenos' y 'malos' clientes potenciales.

\section{f) Árboles de Decisión}

La principal ventaja de esta metodología es que no está sujeta a supuestos estadísticos referentes a distribuciones o formas funcionales. Aunque conllevan una comprensión interna difícil sobre su funcionamiento, presentan relaciones visuales entre las variables, los grupos de la variable respuesta y el riesgo; por ello, este método es muy usado en el credit scoring. Los algoritmos más comunes para construir los árboles de decisión son el ID3, C4.5 y C5. En cada uno de ellos se persigue la separación óptima en la muestra, de tal modo que los grupos de la variable respuesta ofrecen distintos perfiles de riesgo.

La aportación de Breiman, Friedman, Olshen y Stone (1984) fue determinante para el desarrollo de otros trabajos utilizando esta técnica. Entre ellos, Makowski (1985), Coffman (1986) y Carter y Catlett (1987) aplicaron modelos de árboles de decisión para la clasificación de clientes en términos de credit scoring. Boyle, Crook, Hamilton y Thomas (1992) realizaron un estudio comparado de esta metodología con el análisis discriminante, confrontando así una técnica paramétrica ante otra no paramétrica.

Como conclusión general a los modelos de credit scoring para la banca, podemos afirmar que las variables independientes que explican la variable respuesta (no pago del crédito) suelen tener un corte más cuantitativo que cualitativo. Véase el estudio de Allen, Dellong y Saunders (2004) en el que se exponen los modelos más representativos de credit scoring surgidos a partir de los autores precursores de cada técnica. Como se puede comprobar en dicha publicación, las variables explicativas resultantes más significativas de cada uno de estos modelos tienen un carácter cuantitativo sobre la información económica y financiera en la que, sobre todo, resultan ratios formados a partir de dicha información.

\section{Modelos de Credit Scoring en las Instituciones de Microfinanzas}

El riesgo de crédito en las entidades de microfinanzas se manifiesta de la misma forma que en el ámbito bancario. Desde sus orígenes, la actividad microfinanciera ha requerido sistemas de gestión adecuados para minimizar los costes. Las limitaciones e inconvenientes en la elaboración de sistemas de calificación estadística del cliente potencial plantean dificultades a la hora de construirlos, hecho que se refleja en la escasa literatura existente hasta la fecha sobre modelos de credit scoring para las IMFs. Hay autores que discuten sobre la conveniencia o no y sobre la posibilidad de éxito de los modelos de crédito scoring para las microfinanzas. Ese es el caso de Dennis (1995), Kulkosky (1996) y Schreiner (2002), quienes aportan las limitaciones, ventajas e inconvenientes de los modelos de evaluación del riesgo de crédito en las microfinanzas.

Los modelos de credit scoring en microfinanzas publicados hasta la actualidad generalmente están diseñados en las regiones de América Latina y del Sur de África, como lo evidencian Vogelgesang (2003) y Kleimeier y Dinh (2007) en sus investigaciones. Por su parte, Schreiner (2002) afirma que los modelos planteados por Sharma y Zeller (1997), Reinke (1998) y Zeller (1998) no son estadísticamente válidos, al tiempo que indica que los modelos de Sharma y Zeller (1997) y de Zeller (1998) no son viables por estar construidos sobre grupos mancomunados, argumentando que el credit scoring no tiene validez para préstamos en grupo.

El primer modelo de credit scoring para microfinanzas que conocemos fue desarrollado por Viganò (1993) para una institución de microfinanzas de Burkina Faso. Sobre una muestra de 100 microcréditos, y contando con 53 variables iniciales, Viganò utilizó el análisis discriminante para la elaboración del modelo. Como consecuencia del reducido tamaño muestral, el autor tuvo que reagrupar las 53 variables en 13 factores, aunque ello complica la identificación de las características explicativas del no pago del microcrédito.

Sharma y Zeller (1997) elaboraron un estudio similar para una IMF de Bangladesh, donde contaron con 868 
créditos para el análisis. Tras aplicar una metodología Tobit, basada en estimación por máximo-verosimilitud, los autores obtuvieron 5 variables significativas de las 18 que inicialmente contaban con información. Por su parte, Reinke (1998) utilizó un modelo Probit para la elaboración y construcción de un credit scoring para una entidad de microcrédito de Sudáfrica, en el que aceptó las 8 variables explicativas disponibles para una muestra de 1,641 microcréditos. Zeller (1998) diseñó un modelo estadístico de clasificación del cliente para una institución de microfinanzas de Madagascar, también con metodología Tobit. El autor disponía de una muestra de 168 observaciones, incorporando 7 de las 18 variables que tenía por crédito.

Schreiner (1999), con una muestra de 39,956 microcréditos, desarrolló un modelo en el que empleó la regresión logística binaria en clientes de Bancosol (Bolivia), y en el que incluyó las nueve variables independientes disponibles. Dichas variables fueron resumidas en 1) experiencia como prestatario; 2) historial de morosidad; 3 ) género; 4) sector de actividad; 5) cantidad desembolsada; 6) garantías; 7) sucursales; 8) oficiales de crédito; y, 9) la fecha del desembolso. También para Bolivia, Vogelgesang (2003) formuló dos aplicaciones estadísticas para dos entidades de 8.002 y 5.956 casos respectivamente, mediante un modelo de utilidad aleatoria bajo los supuestos de Greene (1992). En la región de Latinoamérica, Miller y Rojas (2005) formularon un credit scoring de Pymes de México y Colombia respectivamente, mientras que Milena, Miller y Simbaqueba (2005) hicieron lo mismo para microfinancieras de Nicaragua. En Mali, Diallo (2006) volvió a emplear la regresión logística para una muestra de 269 créditos de una entidad microbancaria del país. Diallo solo obtuvo 5 variables significativas en su modelo.

Por último Kleimeier y Dinh (2007) construyeron una aplicación de credit scoring para la banca minorista de Vietnam mediante el uso de la regresión logística binaria. La muestra para el modelo estaba conformada por 56.037 créditos de todo tipo (microempresas, consumo, hipotecarios, personales). Obtuvieron 17 variables significativas de 22 variables explicativas, aplicando una combinación adecuada de los conceptos de sensibilidad y especificidad ${ }^{1}$ sobre el porcentaje de aciertos.

\section{MODELO DE CREDIT SCORING PARA INSTITUCIONES DE MICROFINANZAS}

\section{Justificación y objetivos del estudio}

En los países desarrollados, las entidades financieras apoyan su decisión de concesión de un crédito en modelos matemáticos que predicen su probabilidad de impago a partir del comportamiento pasado de una cartera, con el objetivo de mejorar la eficiencia y la cuenta de resultados. La cuestión es si un procedimiento similar es compatible con el negocio de las entidades de microfinanzas. La escasa literatura al respecto sugiere que nos encontramos ante un campo poco explorado. Por otra parte, las limitaciones existentes han conducido a modelos y aplicaciones de la medición del riesgo de impago en microfinanzas que no son adecuadas para la toma de decisiones, ya que, como apunta Schreiner (2000), los modelos de credit scoring en microfinanzas apoyan la decisión del analista de créditos, pero no la sustituye.

Entendemos que este aspecto es muy importante en el contexto actual de incertidumbre en las IMFs, lo que justifica una investigación dirigida al análisis del riesgo de impago en estas instituciones. En efecto, el objetivo de nuestra investigación consiste en diseñar un modelo de credit scoring que supere las limitaciones anteriormente comentadas. El modelo econométrico que pretendemos construir está basado en el conocimiento de las características de los créditos en el momento de su desembolso y su comportamiento de pago después del desembolso. Tiene, por tanto, una doble finalidad: por una parte es explicativa, y por otra predictiva. Es decir, nos proponemos estimar la probabilidad de impago de futuros créditos potenciales antes del desembolso

1 Se entiende por sensibilidad la probabilidad de clasificar correctamente a un cliente que atiende el pago de sus deudas. Por el contrario, la especificidad es la probabilidad de clasificar correctamente a un cliente fallido 
por parte de la Edpyme. No obstante lo antedicho y a partir del modelo desarrollado, esta investigación tiene como objetivo calcular la prima de riesgo, la pérdida esperada, la pérdida inesperada o capital requerido, la tasa de interés adecuada según el riesgo del cliente, y el cálculo de la rentabilidad ajustada al riesgo.

\section{Las entidades de desarrollo de la pequeña y micro empresa (Edpymes)}

La cartera de microcréditos utilizada en la investigación corresponde a la Edpyme Proempresa, entidad de microfinanzas del sistema financiero peruano. Actualmente, las IMFs que operan en el Perú se clasifican en tres grupos:

a) Cajas Municipales de Ahorro y Crédito (CMAC);

b) Cajas Rurales de Ahorro y Crédito (CRAC);

c) Entidades de Desarrollo de la Pequeña y Micro Empresa (EDPYME).

La cobertura de servicios financieros de las entidades financieras se vincula directamente con el tamaño de la empresa con la que se relaciona. Así, la banca centra sus esfuerzos principalmente en corporaciones empresariales $y$, en gran medida, en las empresas medianas. Por otra parte, las entidades de microfinanzas se acercan a aquellos sectores de menor riqueza y a aquellas empresas de menor dimensión, donde la obtención de una línea de crédito presenta muchos más obstáculos.

Según el marco legal peruano², las Edpymes están autorizadas a conceder créditos directos a corto, mediano y largo plazo; otorgar avales, fianzas y otros tipos de garantías; descontar letras de cambio y pagarés. Reciben, también, líneas de financiación procedentes de instituciones de cooperación internacional, organismos multilaterales, empresas o entidades financieras y de la

2 Resolución de la SBS (Superintendencia en Banca y Seguros) $n^{\circ}$ 847-94 del 23 de diciembre de 1994, Resolución de la SBS no 259-95 del 28 de marzo de 1995 (Reglamento) y artículo 10 de la Ley General de Instituciones Bancarias, Financieras y de Seguros (Decreto Legislativo ${ }^{\circ} 770$ del $1^{\circ}$ de abril de 1993).
Corporación Financiera de Desarrollo (COFIDE). No obstante, las Edpymes, a diferencia de las otras empresas del sistema financiero, no están inicialmente autorizadas a captar ahorros del público, sino que solo pueden hacerlo después de tres años de funcionamiento y bajo el cumplimiento de ciertos requisitos. De acuerdo con lo antedicho, entonces, se percibe que son entidades que se ajustan a las características económicas y sociales de las IMFs.

Según puede apreciarse en la Tabla 1, las CMACs son las IMFs que contaban con un mayor número de créditos concedidos a Pymes entre el 2001 al $2005^{3}$. Por su parte, las Edpymes, entidades de reciente creación y regulación por la SBS, han escalado en el ranking de la colocación de créditos a Pymes, superando incluso a las CRACs.

Tabla 1. Saldo medio mensual de créditos a pymes en las IMFs

\begin{tabular}{lc}
\hline \multicolumn{1}{c}{ Empresas } & Saldo medio mensual \\
\hline Total CMAC & 750,688 \\
Total CRAC & 15,779 \\
Total EDPYMES & 201,648 \\
Total & $1 ' 104,116$ \\
\hline
\end{tabular}

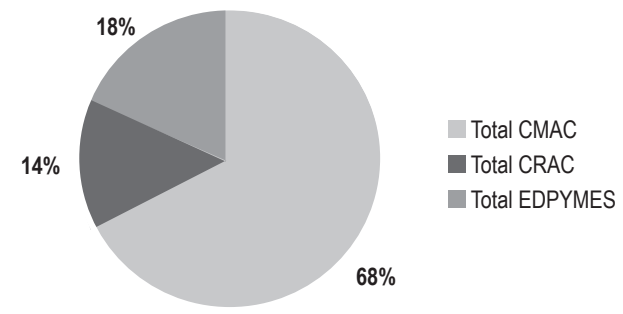

Fuente: Información recabada de la SBS.

Figura 1. Distribución del microcrédito en el sistema microfinanciero peruano.

3 La cartera de préstamos de nuestro estudio contiene créditos concedidos a Pymes durante los años comprendidos entre 1997 y 2005. Sin embargo, únicamente hemos podido disponer datos de la SBS a partir del año 2001. 
Por otra parte, la Tabla 2 nos muestra la distribución del saldo medio mensual de créditos a Pymes entre las Edpymes del sistema financiero peruano. Como puede observarse, la Edpymes Proempresa mantiene una buena posición estratégica respecto del resto de empresas del mismo sector y naturaleza; en tal sentido, colegimos que su cartera de microcréditos es representativa.

Tabla 2. Saldo medio mensual de créditos a Pymes en las Edpymes

\begin{tabular}{lc}
\hline \multicolumn{1}{c}{ Empresas } & Saldo Medio mensual \\
\hline EDPYME Edyficar & 88,803 \\
EDPYME Confianza & 20,646 \\
EDPYME Proempresa & 20,568 \\
EDPYME Crear Arequipa & 18,197 \\
EDPYME Crear Tacna & 13,007 \\
EDPYME Raíz & 11,350 \\
EDPYME Nueva Visión & 5,726 \\
EDPYME Crear Trujillo & 5,154 \\
EDPYME Pro Negocios & 4,912 \\
EDPYME Credivisión & 4,555 \\
EDPYME Crear Cusco & 4,293 \\
EDPYME Alternativa & 3,106 \\
EDPYME Solidaridad & 3,036 \\
EDPYME Efectiva & 1,109 \\
\hline TOTAL EDPYMES & 201,648 \\
\hline
\end{tabular}

\section{Limitaciones de los Credit Scoring en microfinanzas}

La cuestión fundamental que actualmente se plantea en los trabajos de investigación sobre credit scoring es si los modelos estadísticos de riesgo de crédito son aplicables o no a las instituciones de microfinanzas. Schreiner (2000) indica que aunque la calificación es menos enérgica en los países pobres que en los países ricos, y aunque la calificación en las microfinanzas no reemplazará el conocimiento personal del carácter por parte de los analistas de crédito y de los grupos de crédito, la calificación puede mejorar las estimaciones del riesgo y disminuir los costos. Por tanto, indica el autor, la calificación complementa, pero no sustituye, las tecnologías que actualmente se aplican en las microfinanzas.

La construcción de un modelo de credit scoring para las instituciones de microfinanzas implica una serie de limitaciones y desventajas que incrementan la dificultad para llegar a unos resultados razonables. A continuación exponemos algunas de esas limitaciones que, en algunos casos, otros autores han revelado en el intento de consecución de un modelo de scoring para las microfinanzas, y en otros hemos podido detectar al elaborar el presente trabajo.

a) El historial de crédito

El negocio de las IMFs se ha caracterizado por tener historiales de créditos irregulares, incompletos y con un número escaso de observaciones sobre el préstamo. Si lo que pretendemos es analizar el comportamiento de pago de un cliente de microcrédito, se requiere una base de datos amplia que recoja la historia de los préstamos que han resultado impagados en algunas de sus cuotas desde su concesión hasta su fecha de vencimiento. Por otro lado, las IMFs normalmente no incluyen en sus bases de datos la información referente a los clientes a los que se les denegó el crédito porque, en su momento, no pasaron la evaluación estándar del analista. En consecuencia, solo se podrá contar con la información de aquellas solicitudes de crédito que están en la fase de aprobación.

b) La figura del analista de crédito

A diferencia de las entidades financieras, la elaboración de un modelo de calificación estadística para las microfinanzas requiere la intervención de un analista de crédito en la recopilación y captación de información para el historial de crédito. El analista recopila información de tipo personal y cualitativo sobre el cliente, información que podría indicar factores de riesgo para la devolución del crédito concedido. Sin embargo, este proceso podría estar muy influenciado por la opinión subjetiva de dicho funcionario. 
c) El sistema de información

Los datos recogidos por el analista u oficial de crédito han de ser integrados correctamente en el sistema de información de gestión de la entidad de microfinanzas. Esta labor es delicada y requiere un programador u operario destinado exclusivamente a tal función. La razón es simple: si se cometen errores en esta fase, o se demora la introducción de los datos en un tiempo razonable, el modelo diseñado perdería su efectividad.

\section{Definición de incumplimiento}

El incumplimiento de pago debe definirse con cautela, por lo que es necesario identificar todo atraso que conlleve un coste para la organización. Para ello se han de verificar las siguientes condiciones:

a) El atraso percibido ha de ser real y no estimado, según fechas concretas marcadas en la contratación del crédito, en función del método estipulado para su amortización por las partes contratantes.

b) El atraso ha de producirse en, al menos, una cuota de amortización del microcrédito.

c) El atraso considerado ha de suponer un incremento en el coste para la entidad más que proporcional al habitual en caso de no sucederse esta contingencia. Generalmente, estos incrementos suelen darse en términos de costes administrativos debido al incremento monetario que supone realizar un seguimiento y gestionar el pago de un crédito cuyo reembolso mantiene un retraso considerable.

En el modelo de calificación riesgo de morosidad para los créditos de una organización de microfinanzas en Bolivia, Schreiner (1999) establece el atraso costoso como un "atraso de 15 días o más", sin argumentar los motivos en los que se basa para determinarlo. En nuestro caso, vamos a definir el concepto de atraso en el pago que supone un coste añadido para la organización con un mínimo de 30 días desde el vencimiento de al menos una cuota de amortización del microcrédito concedido a un determinado cliente. Este vencimiento respeta los plazos de atraso que conllevan un coste para la organización. Al mismo tiempo, hemos considerado esta referencia temporal por indicación de la propia organización, corroborada por los informes emitidos por la agencia de rating para microfinanzas MicroRate, que considera como cartera en riesgo aquella que está compuesta por créditos atrasados en más de 30 días junto con los créditos refinanciados ${ }^{4}$.

La variable dependiente en el modelo estadístico es dicótoma con un valor de uno (1) para los créditos impagos en las características anteriormente señaladas y cero (0) para los créditos que resultaron pagados en cada vencimiento.

\section{Selección de la muestra}

La base de datos de la cartera de microcréditos de la Edpyme Proempresa contiene información del comportamiento de pago histórico de los clientes que solicitaron créditos dentro del periodo 1997-2005. En la selección de la muestra utilizada para la elaboración del modelo de credit scoring se ha seguido el siguiente procedimiento:

a) Los datos de la muestra constituyen una fuente de información que son el resultado del proceso que sigue la entidad en la concesión de microcréditos (véase el siguiente acápite Proceso de evaluación del microcrédito). En esta línea, Kim (2005) construye un modelo de riesgo de crédito cuya aplicación estadística depende del proceso de concesión de un crédito agrario.

b) Se ha seleccionado la muestra de modo que el número de casos de crédito impago se aproxime al número de casos que pagan, evitando así el problema de la diferencia de tamaños de grupos que, generalmente, provoca una estimación inexacta al no definirse correctamente los factores de clasificación hacia los grupos previamente definidos (Mures et al., 2005).

4 Se considera crédito refinanciado al financiamiento directo respecto del cual se producen variaciones de plazo y /o cantidad del contrato original y que obedecen a dificultades de pago del deudor. 
c) Se pretende que la muestra aporte la mayor cantidad de información posible del cliente, tanto en términos cuantitativos como cualitativos.

d) Los casos admitidos han de contener toda la información de las variables explicativas. Se han eliminado aquellos casos en los que no se aporta información en una determinada variable que sea analizada y seleccionada para la construcción del modelo.

e) Los datos considerados estarán referenciados al mismo periodo de tiempo, tanto para los casos de impago como para los casos en los que se atiende el pago.

El historial de crédito fue extraído del sistema integrado de gestión de la institución, sistema que guarda información cuantitativa y cualitativa relativa a cada cliente en la fecha de la concesión del microcrédito. Posteriormente, dicha información fue descargada a una base de datos en Excel. En dicha base se dispuso de información que no necesariamente constituían variables explicativas, pero era necesaria para la definición de éstas. Una vez seleccionada la muestra, se eliminaron aquellos créditos cuya información relevante estaba incompleta para la selección definitiva de las variables explicativas del modelo. Finalmente, tras un proceso de eliminación de missing value, se construyó una base con una muestra de 5.451 microcréditos que corresponden al periodo comprendido entre los años 1997 y 2005.

Según se indica en la Tabla 3, la muestra fue dividida aleatoriamente en dos submuestras. La intención era realizar una validación a posteriori sobre el modelo de regresión logística estimado, destinando el 75\% de los casos totales a la estimación del modelo estadístico y el $25 \%$ restante a la validación del mismo. La totalidad de los casos que componen las dos muestras son microcréditos concedidos y no rechazados por la entidad. Así, el modelo diseñado asume como fuente de datos la información de prestatarios aceptados según los procesos habituales del manual de procedimientos de gestión del microcrédito de la Edpyme Proempresa.
Tabla 3.

Cartera microcréditos Edpyme Proempresa

\begin{tabular}{ccc}
\hline \multicolumn{3}{c}{ Edpyme Proempresa } \\
Periodo Estudio 1997 / 2005 \\
\hline \multicolumn{3}{c}{ Observaciones (N) } \\
\hline Pagos & Impagos & TOTAL \\
\hline 2,673 & 2,778 & 5,451 \\
\hline \multicolumn{3}{c}{ Muestra Estimación (75\%) } \\
\hline 2,016 & 2,072 & 4,088 \\
\hline \multicolumn{3}{c}{ Muestra Validación $(25 \%)$} \\
\hline 657 & 706 & 1,363 \\
\hline
\end{tabular}

Los créditos considerados en la muestra corresponden a microempresas y Pymes. Son créditos concedidos a personas naturales o jurídicas para la financiación de actividades de producción, comercio o prestación de servicios y cuyos montantes se encuentran dentro de los límites legales establecidos por la normativa SBS para microempresas y Pymes en el periodo de estudio. En este sentido, en la Tabla 4 se puede observar el rango del montante de los microcréditos que componen nuestra cartera, así como los estadísticos descriptivos de la media y desviación típica respecto de esta variable.

Tabla 4.

Estadísticos descriptivos del montante del crédito

\begin{tabular}{ccccc}
\hline & Mínimo & Máximo & Media & Desv. Típica \\
\hline Montante & 60.00 & 3910.00 & 688.20 & 623.93 \\
\hline
\end{tabular}

Si los préstamos otorgados se dan a personas naturales, éstas deberán tener como fuente principal de ingresos la realización de actividades empresariales. Por este motivo, los créditos otorgados para consumo o los préstamos hipotecarios no formarán parte de la muestra.

El proceso de evaluación del microcrédito. Las variables explicativas

Una vez definido el objetivo de la investigación, conceptualizada la variable dependiente (default) y determinada la muestra, la fase siguiente consiste en seleccionar las 
variables explicativas de la capacidad que un cliente posee para atender la devolución del microcrédito a partir del procedimiento de evaluación y concesión de un microcrédito.

La construcción del modelo de credit scoring requiere un análisis previo de las fases que tiene el proceso de promoción, evaluación y concesión de un microcrédito en la Edpyme Proempresa con una doble función, que a continuación se explica:

a) En primer lugar, el proceso de evaluación del microcrédito indicará cuáles son las variables explicativas consideradas para la construcción de la aplicación de credit scoring

b) En segundo lugar, el proceso servirá como base para la realización del modelo estadístico en cuanto a la inclusión de las variables independientes, según vayan interviniendo, en las fases específicas de la concesión del microcrédito.

Diversas normas se desprenden del tratamiento de la información de los historiales de créditos de las entidades supervisadas por la SBS que afectan directamente a la metodología de concesión del crédito para estas entidades. En este sentido, Alvarado (2001) describe el método de otorgamiento de un crédito para una CMAC y para una Edpyme. En líneas generales, ambas coinciden en gran medida al método que proponemos para la evaluación y concesión de un microcrédito. Por este motivo, consideramos que se trata de una metodología que responde a la práctica del mercado en el sistema financiero peruano.

En la Figura 1 se recogen las fases susceptibles de análisis desde el momento en que el crédito es promocionado hasta que éste es concedido y desembolsado. Al mismo tiempo, se considera el seguimiento que el analista realiza con los créditos que presentan problemas de reembolso.

El proceso requiere que se determinen en cada una de las fases las variables explicativas (del cliente, de la operación y macroeconómicas) definidas en la Tabla 5 para su inclusión en el modelo matemático, tal como se detalla a continuación:
1) Fase 1: Investigación de mercado y promoción del crédito

El proceso comienza con la identificación de la sucursal o agencia a partir de la cual se realizará la investigación de mercado y posterior promoción del crédito. La entidad establece como objetivo determinar el procedimiento para informar a las personas naturales y jurídicas sobre los microcréditos que la Edpyme Proempresa brinda con el propósito de ofertar una financiación que satisfaga las necesidades del cliente. La primera variable explicativa que se debe considerar en el análisis es la ZONA.

2) Fase 2: Informes de créditos para clientes nuevos o recurrentes

La entidad de microfinanzas, representada por el analista de crédito, determina el procedimiento para informar y orientar al cliente sobre los productos y servicios de créditos que ofrece la institución. Debido al desconocimiento que numerosos clientes manifiestan sobre el acceso al crédito, la entidad de microfinanzas realiza labores de asesoría financiera para el cliente y, en ocasiones, también desempeñan funciones de asesoría de inversiones y gestión de activos y pasivos. Todo esto se ofrece, junto con la información sobre los productos ofertados, con el objeto de que los analistas de crédito presenten al cliente el producto financiero más conveniente. En esta fase se comprueba si el prestatario es ya cliente de la institución. Por tanto, las variables que se deben tomar en cuenta en esta fase son: ANTIGUO, CRED_CON, CRED_CON 2 y CRED DENEG.

\section{3) Fase 3: Evaluación del expediente de crédito}

La actividad de esta fase del proceso la desarrolla el analista de crédito, quien determina un procedimiento para la evaluación de los expedientes de créditos a ser presentados al Comité de Créditos, que aprueba o rechaza la solicitud. Las etapas que el analista de crédito sigue para realizar este cometido son tres: 


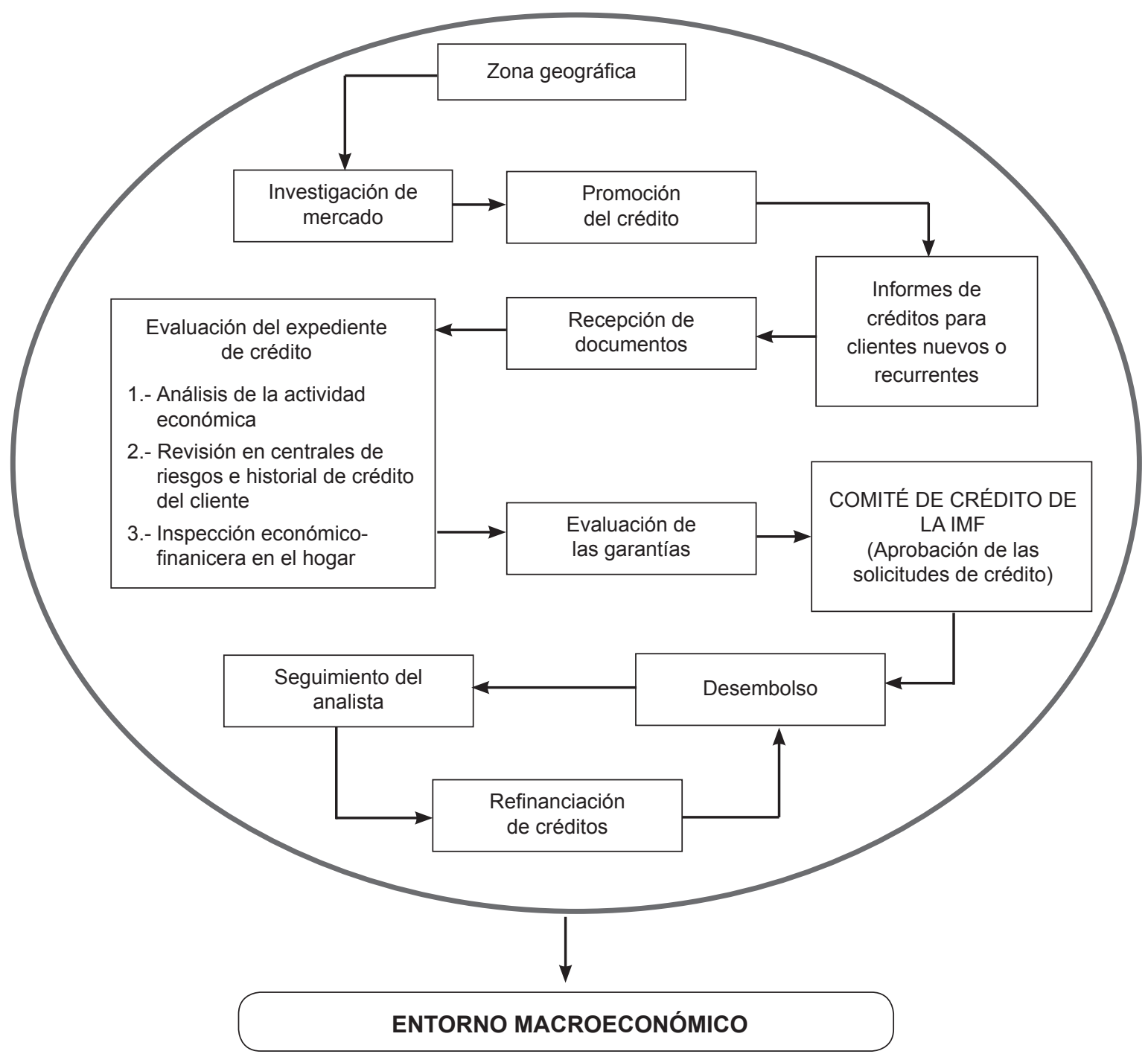

Figura 1. Proceso de promoción, evaluación y concesión de un microcrédito

a) Análisis de la actividad económica desarrollada por el cliente. Las variables subyacentes de esta acción son SECTOR y DEST_CRED.

b) Revisión y comprobación del comportamiento de pago del cliente en centrales de riesgo e historiales de crédito. Aquí, serán consideradas las variables CLAS ENT, CUOT TOT, CUOT_MORA, MEDIA_MORA y MORA MAYOR para su inclusión en el modelo.

c) Inspección económico-financiera de la microempresa. En la mayoría de los créditos, el cliente desarrolla la actividad principal en el seno de la unidad familiar, donde se comprueba si posee casi todo su patrimonio. En este contexto, el analista de crédito visita al prestatario recogiendo la información patrimonial (ratios económicos y financieros $\left(\mathrm{R}_{\mathrm{n}}\right)$ ) y la información personal del solicitante (SEXO, EDAD, E_CIVIL y SIT_LAB).

4) Fase 4: Evaluación de las garantías Como último paso, previo a la entrega del expediente a los Comités de Créditos correspon- 
dientes, el analista de crédito junto con el Jefe de Asesoría Legal de la institución, determina el procedimiento para evaluar las garantías que el solicitante ofrece. En esta fase se incluye la variable GARANT.

5) Fase 5: Aprobación de la solicitud de crédito En este paso el analista de crédito y la gerencia de la entidad deciden aprobar o rechazar los expedientes de crédito presentados. Si la solicitud del préstamo se aprueba, las variables que se toman en cuenta para el modelo estadístico serán: TIPO_OPER, MONEDA, DURACION, INT_MENS, MONTO, MONTO_RECH, y VTOCRED_SBS.

6) Fase 6: Variables Macroeconómicas

Para la elaboración de un modelo de credit scoring se debe tomar en cuenta la influencia del ciclo económico considerando las variables macroeconómicas propuestas en este trabajo según su tasa de variación anualizada. Así, el último bloque de variables que se deben incluir en el modelo son PIB, IPC, IE, TC, TI, IGB, AGUA, LUZ, TFNO.

Las variables independientes se derivan del historial de crédito de la entidad, tomando en cuenta la experiencia del analista y la normativa vigente de la Superintendencia de Banca y Seguros del Perú Con estas variables, el modelo explicará la variable dependiente referente al comportamiento de pago del cliente. Además, el modelo de regresión logística binaria permitirá medir la probabilidad de que un crédito incurra en incumplimiento.

Una contribución importante del presente trabajo es la definición de los signos esperados (positivo o negativo) de los estimadores para cada variable explicativa, definición condicionada al proceso de evaluación y concesión del microcrédito. Tal como está diseñada la variable de la Fase 1, su estimador llevará un signo negativo si se considera que los clientes que viven y desarrollan su actividad productiva en una zona céntrica generalmente presentan menos riesgos que los clientes ubicados en una zona rural.
Relativas a la Fase 2, pertinentes a la antigüedad del cliente como prestatario en la IMF, se espera un signo negativo en el estimador siempre y cuando dicho cliente presente un historial en el que haya respondido correctamente al pago de créditos anteriores y al que le han seguido depositando la confianza otorgándole nuevos créditos. El estimador relativo a créditos anteriores concedidos al cliente y a créditos concedidos en el año que precede a la solicitud también llevará un signo negativo por las mismas razones que la variable anterior. Por último, en cuanto a los créditos previos denegados al cliente, es factible ver un signo positivo en el estimador cuando el pasado de un solicitante ha estado más influenciado por el riesgo de incumplimiento de pago.

En la Fase 3A no se adopta un criterio fijo en el momento de establecer el sector de actividad que implique un mayor riesgo. Sobre cuál es el destino del microcrédito, se propone un signo negativo si el crédito es destinado a la adquisición de un activo fijo que implicaría mayor riesgo que el de un crédito destinado a capital de trabajo; en ese sentido, el proceso de recuperación del activo fijo a través de la amortización técnica conlleva una mayor duración en el tiempo.

Respecto de la Fase 3B, por la forma en que está diseñada la variable que hace referencia a la clasificación del cliente según la Edpyme Proempresa, la expectativa es que un cliente clasificado como "normal" presente menor riesgo que un cliente que haya sufrido contratiempos con su plan de pago. Por su parte, la variable relativa al número de cuotas pagadas registradas en el historial de crédito deberá presentar un signo negativo en un cliente que ostenta mayor número de cuotas, pues supone que, en términos medios, dicho cliente ha demostrado su puntualidad como prestatario. Inversamente, en cuanto al número total de cuotas morosas, la cifra promedio (días) de la morosidad del cliente y el número mayor de días del incumplimiento que presenta se indicará con un signo positivo en los estimadores del modelo por razones obvias. En efecto, un prestatario que presente una cifra alta de cualquiera de estas tres variables indica que ha tenido mayores dificultades para devolver el crédito. 


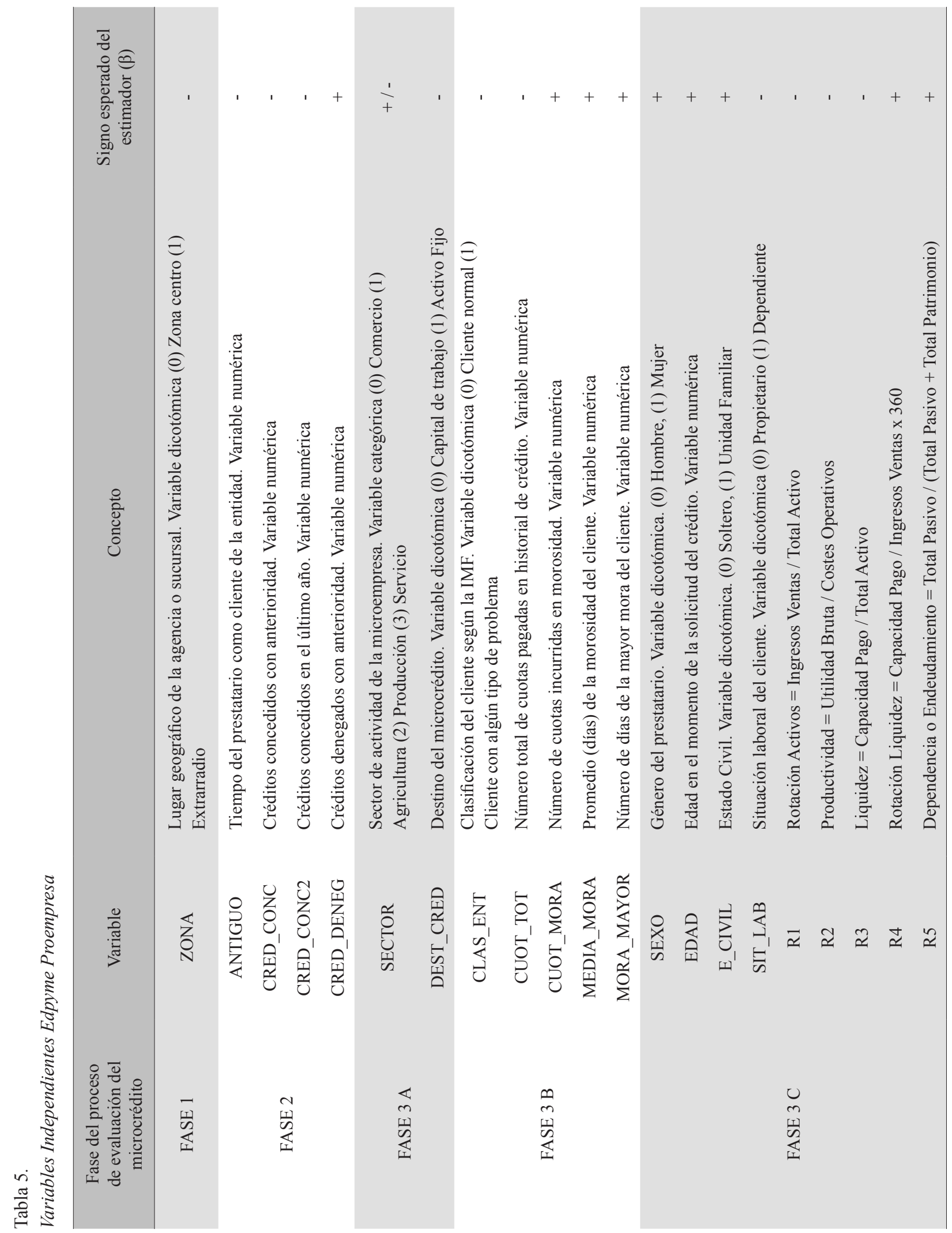




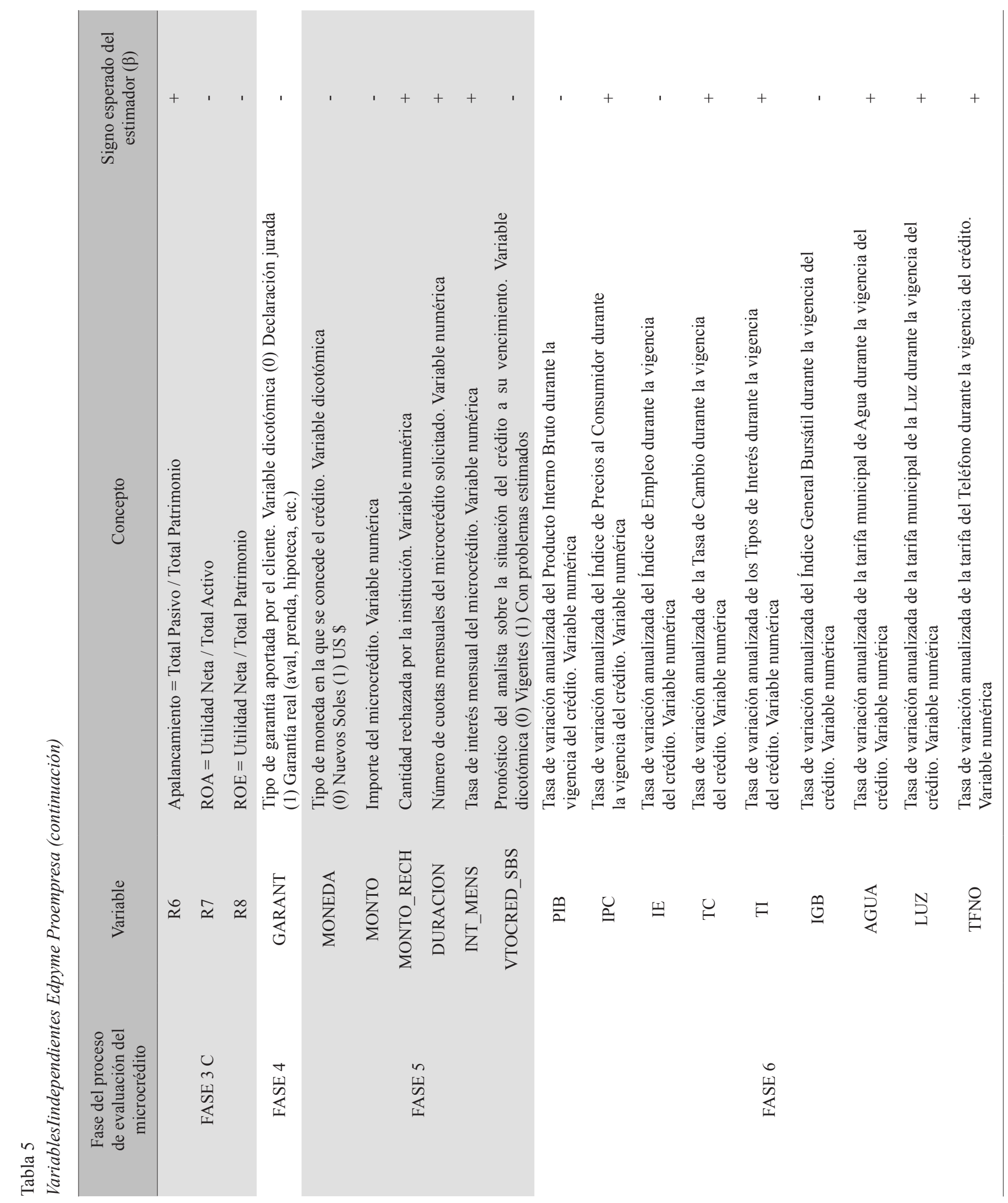


En la Fase $3 \mathrm{C}$, previa a la inspección económicofinanciera de la microempresa o Pyme, el analista de crédito solicitará al cliente su información personal. En microfinanzas en general, las mujeres son mejores pagadoras que los hombres, ya que ellas asumen mayor responsabilidad en el ámbito familiar, hecho que se traslada al comportamiento de pago de un crédito. Por este motivo, se considera un signo positivo para la variable "Género" cuando el sujeto es femenino. El signo positivo para el estimador de la variable "Edad" se coloca cuando un cliente presenta menos edad, pues se considera que posee mayor capacidad en el desempeño de su actividad económica y en el pago de sus deudas con terceros que un cliente de mayor edad. La variable que indica el estado civil del cliente se ve afectada ya que el riesgo de los clientes solteros es mayor que el de los clientes pertenecientes al grupo de los que asumen la responsabilidad de una unidad familiar. En efecto, la responsabilidad familiar y del hogar hace que, por lo general, se traduzca en un mejor comportamiento de pago de las deudas y, por tanto, el signo esperado del estimador estadístico sería positivo. La variable relativa a la situación laboral del prestatario es negativa cuando los clientes que llevan a cabo la actividad económica presenten una situación consolidada. Es decir, el micronegocio en actividad ofrece mayor probabilidad de pago que los clientes que, trabajando en el pasado por cuenta ajena, obtienen un microcrédito para la creación de una microempresa. Por último, el signo esperado de los estimadores de los ratios de patrimonio, liquidez, endeudamiento y rentabilidad han sido contrastados con los modelos de credit scoring para la banca comercial en la literatura correspondiente.

Las garantías aportadas por el cliente en la solicitud del crédito constituyen la variable de la Fase 4. En microfinanzas, la consideración de las garantías en el microcrédito es distinta a las propuestas para los créditos de las grandes corporaciones bancarias. En el ámbito del microcrédito, la reputación o solvencia moral del cliente viene a ser la principal garantía solicitada y aportada. Así, la Edpyme Proempresa generalmente solicita una declaración jurada de bienes a los prestatarios que no suelen tener problemas importantes en el cumplimiento de sus obligaciones de pago. Por el contrario, los clientes que tienen un historial de morosidad en el pago de sus cuotas requieren presentar garantías reales. Por tanto, se espera un signo negativo en el estimador de la variable por la forma en que ha sido diseñada.

En la Fase 5 se indica la moneda en que el microcrédito ha sido concedido. A un microcrédito propuesto en moneda local no le afecta el riesgo cambiario como sí podría a un préstamo en moneda extranjera; por tanto, es conveniente esperar un signo negativo en este estimador. Por un lado, la solicitud de un monto elevado, dentro de los límites impuestos al microcrédito, se acepta si el cliente ha demostrado ser un buen pagador en créditos anteriores. Por otro lado, se deduce que un crédito mayor, por regla general, se concede si las microempresas demuestran estar más asentadas y seguras en los sectores económicos en que se desarrollan. Por tanto, se espera un signo negativo en el estimador de la variable "Monto" cuando se considera que un crédito mayor debe ser menos arriesgado que otro crédito de menor cuantía. Con referencia a la cantidad del préstamo, si éste es rechazado tras la evaluación crediticia, cuanto mayor sea, mayor es el riesgo de impago que la entidad ha percibido en el cliente. En consecuencia, entendemos que el signo del estimador de esta variable debe ser positivo.

En la misma Fase 5, cuanto mayor sea la duración del crédito, mayor deberá ser la probabilidad del riesgo de no pago. Por esta razón, esta variable puede presentar un estimador positivo. La siguiente variable de esta fase es el "tipo de interés". Cuanto mayor sea la tasa de interés de la operación, mayores serán las dificultades que el cliente tendrá para reembolsar el crédito, por lo que se considerará un estimador positivo en esta variable. Por último, cierra las variables de la operación de préstamo el pronóstico del analista sobre la situación del crédito a su vencimiento. Un pronóstico favorable indica que la probabilidad de no pago se reduce, por tanto el signo de esta variable será negativo.

Mención especial merecen las variables que añaden información sobre el ciclo económico ${ }^{5}$. Aún siendo un

5 Según Mester (1997), la ausencia de las variables macroeconómicas ha supuesto una fuerte limitación a los modelos de 
país en vías de desarrollo, el Perú mantiene una economía global como un sistema integrado en el contexto económico internacional. Por tanto, la actividad económica del país se ve influenciada, de alguna manera, por los ciclos económicos a nivel mundial.

Inicialmente, la base de datos de la Edpyme Proempresa no contenía información del ciclo económico. Teniendo en cuenta el corto plazo en términos medios que los microcréditos tienen, para el modelo se consideró una tasa de variación en cada una de las variables macroeconómicas al momento en que se concedía el crédito, según la duración del préstamo. Para tal efecto, se tomó la información de dos instituciones reguladoras peruanas, el Instituto Nacional de Estadística e Informática (INEI) y de la Superintendencia de Banca y Seguros (SBS), para las variables macroeconómicas seleccionadas durante el período 1997-2005.

La siguiente expresión muestra cómo fueron calculadas las tasas de variación de cada una de las variables macroeconómicas consideradas en la construcción del modelo de credit scoring:

$$
\Delta V M_{i, j}=\frac{V M_{i+j}-V M_{i}}{V M_{i}}
$$

siendo

$$
\begin{array}{ll}
\Delta V M_{i, j} & : \text { tasa de variación de la variable } \\
& \text { macroeconómica considerada } \\
V M & : \text { variable macroeconómica considerada } \\
i & : \text { momento de la concesión del crédito } \\
j & : \text { duración del crédito }
\end{array}
$$

Con este cálculo se identificó la evolución de cada variable del ciclo económico en el tiempo transcurrido

Credit Scoring tradicionales, en términos de adaptación de éstos a la realidad económica. Ya lo consideraron Dimitras et al. (1996) al afirmar que la entidad financiera se encontraba inmersa en un entorno a la hora de evaluar el riesgo de insolvencia de una firma y que los cambios en el entorno, así como el comportamiento de las variables macroeconómicas nacionales e internacionales, se afianzaban como factores claves que afectaban de forma directa al comportamiento de pago de la empresa. entre la concesión y el vencimiento de cada microcrédito de la cartera. En seguida, con objeto de hacer comparables los resultados, se anualizaron todas las tasas de variación obtenidas en cada una de las variables macroeconómicas.

Al respecto, Greene (1992) empleó un mayor número de características demográficas y socioeconómicas del cliente, así como variables del prestamista y variables demográficas y macroeconómicas de la región en la que la entidad financiera desarrolla su actividad. En esta línea, Belloti y Crook (2007) construyeron una aplicación de scoring utilizando la regresión logística en la que intentaron analizar el impacto que tenía incluir una serie de variables que reflejaban las características del ciclo macroeconómico. Inicialmente, los autores elaboraron el modelo sin las variables macroeconómicas, contando tan solo con variables del prestatario y de la operación de préstamo, casi todas ellas dummy, y que ya estaban contrastadas en la literatura. Seguidamente, y sobre este mismo planteamiento, procedieron a la incorporación de variables relacionadas con el ciclo económico, tales como tipos de interés, índice general bursátil, producto interno bruto, tasa de desempleo, precio de la vivienda, índice de precios y un ratio de riqueza incluyendo los valores de renta fija. La investigación concluye que la incorporación de estas variables mejora la capacidad predictiva del modelo, en el que el tipo de interés es la variable que mayor impacto refleja en análisis.

\section{Modelo de Regresión Logística Binaria}

Para plantear un modelo cuya variable respuesta o dependiente sea binaria - ya que las dos situaciones posibles es que el cliente paga (0) o el cliente no paga (1) -, se aplicará un modelo de Regresión Logística Binaria con el objeto de evitar los inconvenientes que presentan los modelos de Regresión Lineal o de Análisis Discriminante. Dentro de las técnicas paramétricas ${ }^{6} \mathrm{de}$

6 Las técnicas paramétricas de credit scoring utilizan una función de distribución o clasificación conocida, al igual que estiman unos parámetros para explicar un determinado suceso, de tal modo que éstos se ajusten a las observaciones de una muestra. Estas técnicas son muy útiles cuando el conjunto de variables siguen una distribución propuesta. 
credit scoring, se eligió la Regresión Logística Binaria como técnica estadística empleada debido a sus mayores ventajas, fundamentalmente por los siguientes motivos:

a) Las propiedades estadísticas son mas adecuadas que las de los modelos lineales en los que, en ocasiones, se obtienen estimadores ineficientes;

b) dadas las características del historial crediticio de la Edpyme Proempresa, donde la información cualitativa complementa la escasez de variables cuantitativas, la Regresión Logística Binaria admite las variables categóricas con mayor flexibilidad que los modelos lineales;

c) permite estimar la probabilidad de impago del crédito según los valores de las variables independiente;

d) determina la influencia de cada variable independiente sobre la variable dependiente (pago o impago) según el OR (Odd Ratio o ventaja). Éste se define como $\exp (\beta)$, donde exp es la base de los logaritmos neperianos (una constante cuyo valor es 2,718), y $\beta$ es el valor del parámetro de regresión de la variable independiente en el modelo. Una OR mayor que 1 indica un aumento en la probabilidad del evento de incumplimiento sobre el hecho de pagar cuando la variable explicativa aumenta en una unidad; inversamente, una OR menor que 1 indica lo contrario.

El modelo de regresión logística puede formularse como:

$$
\log \left(\frac{p}{1-p}\right)=\beta_{0}+\beta_{1} x_{1}+\beta_{2} x_{2}+\ldots . .+\beta_{k} x_{k}
$$

donde $p$ es la probabilidad de ocurrencia del evento de interés, en nuestro caso, impago o default. Debido al valor de las variables independientes, la probabilidad señalada puede ser calculada directamente de la siguiente forma:

$$
p=\frac{e^{Z}}{1+e^{Z}}=\frac{1}{1+e^{-Z}},
$$

siendo

$$
Z=\beta_{0}+\beta_{1} x_{1}+\beta_{2} x_{2}+\ldots . .+\beta_{k} x_{k}
$$

A continuación, se estima la significación estadística de los coeficientes del modelo a través del estadístico de Wald y la bondad del ajuste. Si una variable resulta ser no significativa, se procede inmediatamente a eliminarla del modelo.

\section{RESULTADOS DE LA INVESTIGACIÓN}

\section{Estimación del modelo}

El tratamiento de la base de datos de la cartera de microcréditos de la Edpyme Proempresa, mediante el módulo de Regresión Logística Binaria del software SPSS vs. 15, ofrece como resultado la ecuación de puntuación Z (expresión 5) y la probabilidad de impago $\mathrm{P}$ (expresión 6) para cada uno de los prestatarios de dicha cartera:

$Z=8.703-0.498$ ZONA -1.693 SIT_LAB -3.062

$R 3+1.274$ R5 - 0.070 CRED_CON2 - 1.088 DEST CRED - 1.064 GARANT - 4.356 VTOCRED_SBS + $0.241 \mathrm{TC}$

donde

$\mathrm{P}($ Probabilidad de incumplimiento $)=\frac{1}{1+\mathrm{e}^{-(\mathrm{Z})}}$

Las variables que intervienen en el modelo final de credit scoring se resumen en la Tabla 6. Puede apreciarse que el modelo final queda definido con variables que intervienen en prácticamente todas las fases del proceso de evaluación y concesión del microcrédito, destacando una mayor presencia de éstas en la fase relativa a la inspección económico-financiera de la microempresa.

Al mismo tiempo, se debe destacar que la variación anualizada del tipo de cambio correspondiente al grupo de las variables macroeconómicas forma parte del modelo final de scoring para la Edpyme Proempresa. 
Tabla 6.

Variables independientes Edpyme Proempresa

\begin{tabular}{cc}
\hline $\begin{array}{c}\text { Fase del proceso de evaluación } \\
\text { del microcrédito }\end{array}$ & Variable \\
\hline FASE 1 & ZONA \\
\hline FASE 2 & CRED_CONC2 \\
\hline FASE 3 A & DEST_CRED \\
\hline FASE 3 C & SIT_LAB \\
& R3 \\
FASE 4 5 \\
FASE 5 & GARANT \\
FASE 6 & VTOCRED_SBS \\
\hline
\end{tabular}

Es decir, el ciclo económico influye en la valoración del riesgo de impago de un cliente de microcrédito.

\section{Valoración del modelo}

Para comprobar que el modelo de regresión logística estimado es adecuado para la toma de decisiones se requiere realizar un proceso de valoración concluyente sobre una serie de aspectos. El supuesto de linealidad, tal como se indica en (5), viene impuesto por la propia naturaleza de la fórmula utilizada en la regresión logística.

A partir de los coeficientes del modelo detallado en la Tabla A1 del Anexo A, se observa que dos variables influyen positivamente y siete influyen negativamente en la probabilidad de que un cliente sea moroso. Cuando el signo del estimador es positivo significa que, cuando la variable independiente aumenta en una unidad, el logaritmo de las probabilidades de ser un cliente moroso aumenta en el valor del coeficiente respectivo, y viceversa para los estimadores negativos. En cuanto al impacto estadístico y el signo de los coeficientes, todas las variables presentan signo coherente de influencia en la variable explicativa y $p$-value inferiores al nivel crítico fijado en 0.05 . Por tanto, todas las variables independientes del modelo influyen en el comportamiento de pago del cliente del microcrédito.
Una vez estimado el modelo a partir de los datos observados, el siguiente paso es valorar su importancia global, puesto que la significación individual de cada coeficiente se ha considerado para la inclusión de las variables en la ecuación del modelo, mediante su contribución a la mejora del estadístico $\chi 2$ de dicho modelo. Este análisis estadístico, o test de razón de verosimilitud, se aplica también para valorar la significación del modelo, cuyo valor es de 1848,966, lo que resulta ser trascendental tal como puede observar en la Tabla A2 del Anexo A.

Respecto de la bondad del ajuste, además del estadístico $\chi 2$ del modelo, el software calcula coeficientes similares al $\mathrm{R}^{2}$ computado en regresión lineal. Concretamente se calculan el $\mathrm{R}^{2}$ de Cox y Snell y el $\mathrm{R}^{2}$ de Nagelkerke, cuyos valores respectivos son indicativos de un ajuste aceptable en regresión logística (ver Tabla A3 del Anexo A).

Otro elemento estadístico utilizado para valorar la bondad de ajuste del modelo es la prueba de Hosmer y Lemeshow, para lo cual se agrupan las observaciones para cada uno de los dos grupos definidos por la variable dependiente, según una tabla de contingencia (ver Tabla A4 del Anexo A). La bondad del ajuste determina el grado de semejanza (ajuste) que existe entre los valores observados y los pronosticados por el modelo. Si bien la prueba de Hosmer-Lemeshow no ofrece un resultado satisfactorio -su nivel de significación es bajo-, se debe tomar en cuenta que este dato estadístico solamente brinda pistas sobre la calidad del ajuste del modelo, aunque también se debe recordar que está muy condicionado por el tamaño de la muestra.

Por otra parte, puede apreciarse en la matriz de correlaciones cómo la correlación de las variables explicativas que forman parte del modelo final es reducida (ver Tabla A5 del Anexo A). Ello permite comprobar que no existe relación entre estas variables en cuanto a información se refiere.

A su vez, es interesante valorar la capacidad o eficacia predictiva del modelo, lo que se realiza a partir 
de la tabla de clasificación que se presenta en la Tabla A6 y la Figura A1 del Anexo A en las que se recogen los valores observados para la variable dependiente, distinguiendo entre clientes morosos (1) y no morosos (0), y los valores pronosticados según el modelo estimado. De acuerdo con lo antedicho, el porcentaje correcto de clasificación obtenido para el modelo de credit scoring de la Edpyme Proempresa fue de 78.3\%. Este porcentaje mide la precisión con la que el modelo consigue discriminar entre los casos de ambos grupos (clientes que pagan y clientes morosos).

El punto de corte óptimo que mejor separa los créditos solventes de los morosos se sitúa en 0.58. En efecto, tras el cálculo de los valores de "Sensibilidad" y "Especificidad", este valor constituye la frontera en el que la probabilidad de acierto es mayor, como puede comprobarse en la Tabla B1 del Anexo B. Por otro lado, la Tabla B2 de este mismo anexo muestra el área bajo la curva (curva COR), la cual es $85.9 \%$. Se debe tomar en cuenta que el valor de esta curva oscila entre 0.5 y 1 , en donde 1 indica discriminación perfecta y 0.5 indica que la clasificación que se alcanza no es mejor que la que se obtendría clasificando casos al azar. En esta línea, según puede observarse en la Figura B1 del Anexo B, al aproximarse la curva COR del modelo a la esquina superior izquierda del gráfico se confirma que el modelo dispone de una correcta capacidad de discriminación entre los dos grupos de clientes. En cuanto a la independencia de los residuos del modelo de regresión logística, se asume que la muestra objeto de estudio es aleatoria, por lo que los residuos también mostrarán una pauta similar.

Por último, se debe mencionar que la presencia de valores atípicos para cada una de las variables explicativas del modelo no es significativa. Lo expuesto puede comprobarse a través de la variabilidad reducida de las DFBETAS de las variables explicativas, según se recogen en el Anexo C. En este acápite se mide el impacto en los distintos parámetros del modelo de regresión logística cuando una observación se elimina de la muestra.
Tabla 7.

Porcentaje de acierto en el proceso de validación

\begin{tabular}{lccc}
\hline \multirow{2}{*}{ Observado } & \multicolumn{2}{c}{ Pronosticado } & Porcentaje \\
\cline { 2 - 3 } & Pago (0) & Impago (1) & \\
\hline Pago (0) & 586 & 71 & $89.19 \%$ \\
$\begin{array}{l}\text { Impago (1) } \\
\begin{array}{l}\text { Porcentaje global } \\
\text { de acierto }\end{array}\end{array}$ & 233 & 473 & $67.00 \%$ \\
\hline
\end{tabular}

\section{Validación del modelo}

Si se aplica la fórmula de la probabilidad de incurrir en incumplimiento del modelo estimado (Fórmulas (5) y (6)) a la muestra reservada para la validación, el porcentaje global de acierto es del $77.70 \%$, como se refleja en la Tabla 7.

\section{APLICACIONES DEL MODELO DE CREDIT SCORING}

La principal aplicación del modelo de credit scoring está relacionada, no solo con el proceso de aceptación o rechazo de un crédito a un cliente, sino también con el cálculo del capital necesario para la operación en el marco de los modelos internos de requerimiento de capital de Basilea II. Al mismo tiempo, se determina la tasa de interés que es necesaria aplicar a la operación de crédito (pricing) con el propósito de obtener una rentabilidad ajustada al riesgo de capital (Return On Risk Adjusted Capital) del cliente o de la operación.

Si analizamos el requerimiento de capital de la operación crediticia en el Perú, la Resolución de la Superintendencia de Banca y Seguros (SBS) No. 14.354, del 30 de Octubre de 2009 sobre el Reglamento para el Requerimiento de Patrimonio Efectivo por Riesgo de Crédito, recoge la normativa de adaptación para la implementación de Basilea II en las entidades bancarias y de microfinanzas que son supervisadas. La norma establece, de acuerdo con el Documento de Basilea II de 2004 sobre el requerimiento de capital mínimo 
para cubrir el riesgo de crédito, que el cálculo del capital requerido derivado del riesgo de crédito de la cartera pueda hacerse mediante tres métodos que van de menor a mayor grado de sofisticación, según se detalla a continuación:

a) Método estándar: La entidad no dispone de modelos de calificación y es por tanto el regulador el que aplica "estándares" para la obtención del Capital Mínimo requerido.

b) Método Basado en Calificaciones Internas IRB (Internal Rating Based) Básico: La entidad dispone de modelos de calificación para la estimación de la Probabilidad de Impago (PD), sobre lo cual el regulador aplicará estándares para la obtención de la Severidad (LGD) y Exposición (EAD).

c) Método Basado en Calificaciones Internas IRB (Internal Rating Based) Avanzado: La entidad dispone de Modelos de calificación para la estimación de la probabilidad de Impago (PD), Severidad (LGD) y Exposición (EAD).

Por tanto, es en los modelos de calificación interna donde se necesita el input de la probabilidad de impago que se obtiene del modelo de credit scoring. Para determinar el requerimiento de capital en una operación de crédito, Basilea II considera un cuartil del 99.9\%, con el objeto de que en el cómputo del capital se contemplen el $99.9 \%$ de las situaciones del estado de la economía que tengan influencia en un posible impago del cliente y que el peor resultado, en situación de catástrofe o crisis en la misma, se produzca con una probabilidad del $0.1 \%$. La fórmula para calcular el requerimiento de capital exigido a las entidades de crédito en los modelos internos, según el modelo propuesto por el Comité de Supervisión Bancaria de Basilea para la cartera minorista, es la siguiente ${ }^{7}$ :

$$
\begin{aligned}
& \mathrm{K}=\left[\mathrm{LGD} \cdot \mathrm{N}\left(\frac{\mathrm{G}(\mathrm{PD})+\sqrt{\rho(\mathrm{PD})} \cdot \mathrm{G}(0.999)}{\sqrt{1-\rho(\mathrm{PD})}}\right)-\mathrm{PD} \cdot \mathrm{LGD}\right] \\
& \mathrm{RWA}=\left(\frac{100 \%}{10 \%}\right) \cdot\left[\mathrm{LGD} \cdot \mathrm{N}\left(\frac{\mathrm{G}(\mathrm{PD})+\sqrt{\rho(\mathrm{PD})} \cdot \mathrm{G}(0.999)}{\sqrt{1-\rho(\mathrm{PD})}}\right)-\mathrm{PD} \cdot \mathrm{LGD}\right] \\
& \mathrm{RWA}=\mathrm{K} \cdot 10 \cdot \mathrm{EAD} ; \quad \mathrm{PES}=\mathrm{PD} \cdot \mathrm{LGD} \cdot \mathrm{EAD} \\
& \rho(\mathrm{PD})=0.03 \cdot \frac{1-\mathrm{e}^{-35 \mathrm{PD}}}{1-\mathrm{e}^{-35}}+0.16 \cdot\left(1-\frac{1-\mathrm{e}^{-35 \mathrm{PD}}}{1-\mathrm{e}^{-35}}\right)
\end{aligned}
$$

7 Puede consultarse a Trucharte y Marcelo (2001) para una mayor profundidad de las bases teóricas sobre las que se asienta el modelo propuesto por el Comité de Supervisión Bancaria de Basilea para determinar los requerimientos mínimos de capital exigidos a las entidades de crédito en la normativa de Basilea II. 
donde

K : Requerimiento de capital

PD : Probabilidad de incumplimiento que se obtiene del credit scoring (Probabilty Default)

$\rho(\mathrm{PD}) \quad$ : Coeficiente de correlación

LGD : Severidad o porcentaje de pérdida en el momento del incumplimiento (Loss Given Default)

EAD : Exposición en el momento del incumplimiento

RWA : Activo ponderado por riesgo (RiskWeighted Assets)

PES : Pérdida esperada

G (0,999): Inversa de la Función Distribución Normal Estándar Acumulada $=-3.090$.

G (PD) : Inversa de la Función Distribución Normal Estándar Acumulada en PD

Para analizar el efecto que se da cuando una entidad de microfinanzas maneja un modelo de credit scoring en el marco de la norma de Basilea II, se desarrollará un caso práctico en el que la Edpyme Proempresa concede dos microcréditos. El primero es a un cliente que en el método estándar tiene rating B-; el otro crédito es a un cliente cuyo rating es CCC. Primero se calcularán sus respectivas probabilidades de incumplimiento, según el modelo scoring que se ha obtenido para la cartera de microcréditos de la Edpyme Proempresa (se aplicarán las Fórmulas (5) y (6) a los datos particulares de cada cliente). En segundo lugar, se calculará la rentabilidad ajustada a riesgo (RORAC) de ambos clientes en la normativa de Basilea I y en la de Basilea II según el modelo IRB avanzado. En tercer lugar, se ajustarán las tasas de interés que la IMF debería aplicar a cada cliente para que la rentabilidad ajustada a riesgo de ambos sea igual al 35\%, que es el objetivo mínimo establecido por la institución para este tipo de operaciones. Los datos del caso son los siguientes:

a) Cliente 1: Rating B- (Moderada probabilidad de incumplimiento que se puede ver afectada si cambia el escenario económico financiero a una situación más adversa)

b) Cliente 2: Rating CCC (Existen factores que pueden aumentar significativamente el riesgo de incumplimiento de los términos pactados, aún en el escenario económico corriente al momento de la evaluación)

c) Crédito Microempresa $=1.010 \$ ;$ Plazo $=1$ año; Provisión $=1 \%$

d) Precio crédito (tasa de interés igual en ambos en clientes) $=20.00 \%$ anual

e) Tasa LIBOR a un año $=$ Coste Fondos Ajenos $=$ $5 \%$

f) Costes operativos $=10 \%$ y Tasa impositiva $=$ $35 \%$

g) Objetivo de RORAC $=35 \%$

h) Tasa rentabilidad un Activo Libre de Riesgo $(\mathrm{TLR})=3 \%$ (Bono Gobierno)

i) Aplicación de la Tabla IRB Ponderaciones Riesgo Minorista (Otros)

Si se sustituye en la expresión del modelo de regresión logística los datos de los dos clientes que solicitan un microcrédito (Formulas (5) y (6)), se obtienen las correspondientes probabilidades de impago, según la Tabla 8. En las Tablas 9 y 10 se calculan la rentabilidad que la entidad de microfinanzas obtiene con cada uno de los clientes en el marco de la normativa de Basilea I y la de Basilea II. Además, se calcula la tasa de interés que debería negociarse con cada cliente para conseguir el objetivo de rentabilidad ajustada a riesgo (RORAC), que es del $35 \%$ anual.

Aquí se comprueba que, en el marco de la norma sobre requerimientos de capital de Basilea I, ambos clientes aportan a la Edpyme rentabilidades muy parecidas: cliente de rating B- aporta 44.29\%; el cliente de rating CCC, $46.01 \%$. Sin embargo, la aplicación de la norma Basilea II, mediante el método IRB, permite discriminar en términos de rentabilidad (cliente rating B- $22.40 \%$; cliente CCC -34.32\%). Esto se debe fundamentalmente a que la probabilidad de impago es distinta en ambos 
clientes y, por tanto, también es distinta la ponderación por riesgo; en otras palabras, esto es el activo ponderado por riesgo (RWA) y el importe de capital propio que Proempresa tiene que destinar para cubrir el riesgo de cada cliente.

Si el objetivo de la entidad es conseguir una rentabilidad ajustada a riesgo de capital del $35 \%$ con clientes de microcrédito, entonces Proempresa deberá fijar una tasa de interés del $21.09 \%$ al microcrédito concedido al cliente de rating B- (se le aumenta 1.09\% la tasa inicial del 20\%). Por otro lado, la entidad financiera deberá aplicar una tasa de interés de $28.85 \%$ al microcrédito concedido al cliente de rating CCC (aumentando en $8.85 \%$ la tasa inicial del $20 \%$ ).

Para concluir, se debe tener en cuenta que todo este proceso de aplicación de las normas internacionales sobre requerimientos de capital en instituciones de mi- crofinanzas sujetas a supervisión puede convertirse en un factor de riesgo para la viabilidad y solvencia futura de las mismas por dos motivos: El primero de ellos es que las entidades de microfinanzas con modelos estándar de gestión del riesgo de crédito deberán destinar mayor cantidad de capital que las entidades bancarias que gestionen la cartera a través de un modelo IRB, lo que supone una rentabilidad ajustada riesgo más reducida para las IMFs. Ello significaría que deberán elevar la tasa de interés de los microcréditos. Este hecho evidentemente penalizaría aun más al microempresario e iría en contra de la misión y objetivos de las entidades de microfinanzas. El segundo aspecto a considerar es que en el plazo medio se produciría un trasvase de clientes con rating elevado de las entidades de microfinanzas a las entidades bancarias que cuenten con unidades de microcrédito. En efecto, el requerimiento de capital en las entidades bancarias es menor así como la tasa de interés que se aplica a

Tabla 8.

Probabilidad de impago para un cliente de rating $B$ - $y$ de rating $C C C$

\begin{tabular}{|c|c|c|c|c|}
\hline Datos cliente & Variable & Coef. Logit & Cliente 1 & Cliente 2 \\
\hline RATING & & & B- & $\mathrm{CCC}$ \\
\hline Zona geográfica (0 Céntrica) y (1 Extrarradio) & ZONA & -0.498 & 0 & 0 \\
\hline Situación laboral (0 Propietario) y (1 Dependiente) & $S I T \_L A B$ & -1.693 & 0 & 0 \\
\hline Ratio liquidez & $R 3$ & -3.062 & $52.00 \%$ & $30.00 \%$ \\
\hline Endeudamiento & $R 5$ & 1.274 & $5.00 \%$ & $10.00 \%$ \\
\hline Créditos concedidos con anterioridad & CRED_CON2 & -0.072 & 14 & 7 \\
\hline Destino del crédito (0 Capital Trabajo) y (1 Activo Fijo) & DEST_CRED & -1.088 & 0 & 0 \\
\hline Garantía (0 Sin Garantía) y (1 con Garantía) & GARANT & -1.064 & 0 & 0 \\
\hline $\begin{array}{l}\text { Pronóstico analista crédito a vencimiento (0) Vigentes } \\
\text { (1) Con problemas estimados }\end{array}$ & VTOCRED_SBS & -4.356 & 0 & 0 \\
\hline Variación anual Tasa de Cambio & $T C$ & 0.241 & $5.00 \%$ & $5.00 \%$ \\
\hline Constante & CONSTANTE & 8.703 & & \\
\hline Puntuación scoring (Z) & & & -2.5205 & -1.2792 \\
\hline PD (Probabilidad Default) & & & $7.44 \%$ & $21.77 \%$ \\
\hline
\end{tabular}


Tabla 9.

Rentabilidad del cliente en Basilea I y Basilea II (antes del ajuste de tasas)

\begin{tabular}{|c|c|c|}
\hline Concepto & Cliente 1 & Cliente 2 \\
\hline Tasa interés & $20.00 \%$ & $20.00 \%$ \\
\hline Importe crédito & 1,010 & 1,010 \\
\hline Tasa Libre de Riesgo & $3.00 \%$ & $3.00 \%$ \\
\hline Costes Operativos & $10.00 \%$ & $10.00 \%$ \\
\hline Coste Fondos Ajenos & $5.00 \%$ & $5.00 \%$ \\
\hline Tasa Impuestos & $35.00 \%$ & $35.00 \%$ \\
\hline \multicolumn{3}{|c|}{ Basilea I } \\
\hline Ponderación & $100.00 \%$ & $100.00 \%$ \\
\hline Provisión $(\mathrm{B}-=5 \% ; \mathrm{CCC}=5 \%)$ & 10.10 & 50.50 \\
\hline Coeficiente RRPP Basilea I & $8.00 \%$ & $8.00 \%$ \\
\hline Fondos Propios & 79.99 & 76.76 \\
\hline Fondos Ajenos & 930.01 & 933.24 \\
\hline Ingresos Financieros & 202.00 & 202.00 \\
\hline Costes Financieros & 46.50 & 46.66 \\
\hline Costes Operativos & 101.00 & 101.00 \\
\hline Beneficio de Capital & 0.00 & 0.00 \\
\hline Rentabilidad Cliente & $44.29 \%$ & $46.01 \%$ \\
\hline \multicolumn{3}{|c|}{ Basilea II-IRB } \\
\hline Rating & B- & $\mathrm{CCC}$ \\
\hline PD & $7.44 \%$ & $21.77 \%$ \\
\hline LGD & $45.00 \%$ & $45.00 \%$ \\
\hline Perdida Esperada (EL) & 33.83 & 98.94 \\
\hline Ponderación & $56.0168 \%$ & $82.9780 \%$ \\
\hline RWA & 565.77 & 838.08 \\
\hline Coeficiente Basilea II-IRB & $10.00 \%$ & $10.00 \%$ \\
\hline Fondos Propios & 56.58 & 83.81 \\
\hline Fondos Ajenos & 953.42 & 926.19 \\
\hline Total & $1,010.00$ & $1,010.00$ \\
\hline Superavit/Déficit Fondos Propios & 23.42 & -7.05 \\
\hline Ingresos Financieros & 202.00 & 202.00 \\
\hline Costes Financieros & 47.67 & 46.31 \\
\hline Costes Operativos & 101.00 & 101.00 \\
\hline Beneficio Capital FP & 0.00 & 0.00 \\
\hline Rentabilidad Ajustada a Riesgo (RORAC) & $22.40 \%$ & $-34.32 \%$ \\
\hline Rentabiliad Objetivo & $35.00 \%$ & $35.00 \%$ \\
\hline Precio & $20.00 \%$ & $20.00 \%$ \\
\hline
\end{tabular}


Cuadro 10

Rentabilidad del cliente en Basilea I y Basilea II (después del ajuste de tasas)

\begin{tabular}{|c|c|c|}
\hline Concepto & Cliente 1 & Cliente 2 \\
\hline Tasa interés & $21.09 \%$ & $28.85 \%$ \\
\hline Importe crédito & 1,010 & 1,010 \\
\hline Tasa Libre de Riesgo & $3.00 \%$ & $3.00 \%$ \\
\hline Costes Operativos & $10.00 \%$ & $10.00 \%$ \\
\hline Coste Fondos Ajenos & $5.00 \%$ & $5.00 \%$ \\
\hline Tasa Impuestos & $35.00 \%$ & $35.00 \%$ \\
\hline \multicolumn{3}{|c|}{ Basilea I } \\
\hline Ponderación & $100.00 \%$ & $100.00 \%$ \\
\hline Provisión $(\mathrm{B}-=5 \% ; \mathrm{CCC}=5 \%)$ & 10.10 & 50.50 \\
\hline Coeficiente RRPP Basilea I & $8.00 \%$ & $8.00 \%$ \\
\hline Fondos Propios & 79.99 & 76.76 \\
\hline Fondos Ajenos & 930.01 & 933.24 \\
\hline Ingresos Financieros & 212.97 & 291.38 \\
\hline Costes Financieros & 46.50 & 46.66 \\
\hline Costes Operativos & 101.00 & 101.00 \\
\hline Beneficio de Capital & 0.00 & 0.00 \\
\hline Rentabilidad Cliente & $53.20 \%$ & $121.70 \%$ \\
\hline \multicolumn{3}{|c|}{ Basilea II-IRB } \\
\hline Rating & B- & $\mathrm{CCC}$ \\
\hline $\mathrm{PD}$ & $7.44 \%$ & $21.77 \%$ \\
\hline LGD & $45.00 \%$ & $45.00 \%$ \\
\hline Perdida Esperada (EL) & 33.83 & 98.94 \\
\hline Ponderación & $56.0168 \%$ & $82.9780 \%$ \\
\hline RWA & 565.77 & 838.08 \\
\hline Coeficiente Basilea II-IRB & $10.00 \%$ & $10.00 \%$ \\
\hline Fondos Propios & 56.58 & 83.81 \\
\hline Fondos Ajenos & 953.42 & 926.19 \\
\hline Total & $1,010.00$ & $1,010.00$ \\
\hline Superavit/Déficit Fondos Propios & 23.42 & -7.05 \\
\hline Ingresos Financieros & 212.97 & 291.38 \\
\hline Costes Financieros & 47.67 & 46.31 \\
\hline Costes Operativos & 101.00 & 101.00 \\
\hline Beneficio Capital FP & 0.00 & 0.00 \\
\hline Rentabilidad Ajustada a Riesgo (RORAC) & $35.00 \%$ & $35.00 \%$ \\
\hline Rentabiliad Objetivo & $35.00 \%$ & $35.00 \%$ \\
\hline Precio & $21.09 \%$ & $28.85 \%$ \\
\hline
\end{tabular}




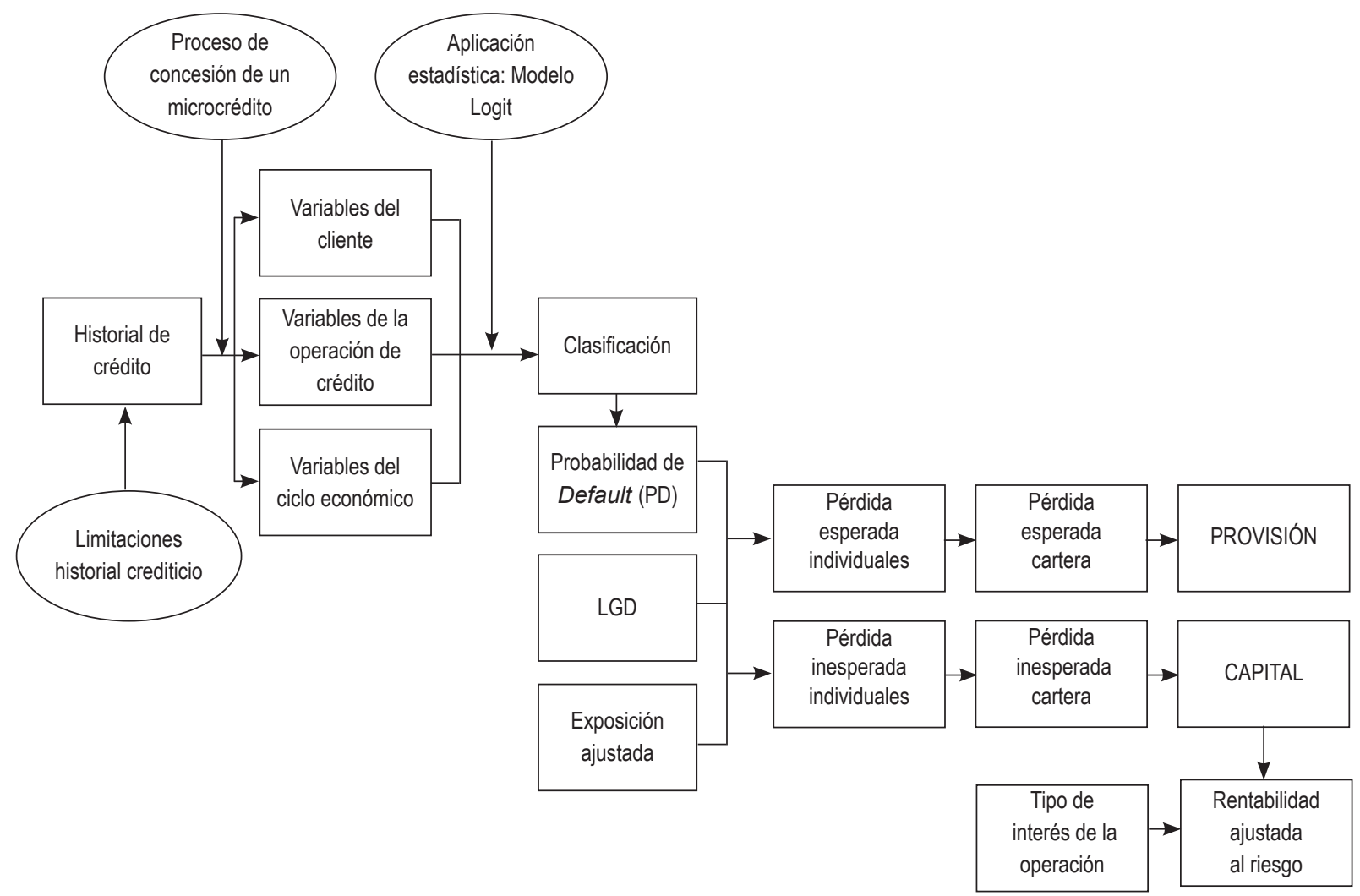

Figura 2. Esquema general de la metodología para el análisis del riesgo de microcrédito

las operaciones. Sin embargo, también puede darse la situación opuesta; es decir, que clientes de bajo rating abandonen la gran banca y se incorporen a la cartera de las IMFs, que es menos costosa en tasas de interés porque mantienen sistemas estándares menos exigentes en requerimientos de capital. La Figura 2 nos muestra el esquema general de la metodología que hemos seguido en el análisis de riesgo de una cartera de microcréditos.

\section{CONCLUSIONES Y FUTURAS LÍNEAS DE INVESTIGACIÓN}

Una revisión bibliográfica de la literatura sobre credit scoring para instituciones de microfinanzas ha permitido comprobar que no existen muchos trabajos sobre el tema. Aproximadamente hay una decena de publicaciones donde se han diseñado modelos de scoring para medir el riesgo de crédito de estas entidades microfinancie- ras. Ello indica que todavía queda amplio margen de investigación en esta línea. En tal sendito, el objetivo principal del presente trabajo es diseñar un modelo de credit scoring para la cartera de microcréditos de la Edpyme Proempresa, entidad supervisada del sistema financiero del Perú. Si bien la muestra de créditos que se tomó para esta investigación es grande en número, se ha comprobado que la información era limitada en numerosos créditos lo que redujo considerablemente el tamaño muestral definitivo para la estimación del modelo y su posterior validación.

Otro aspecto importante fue definir las fases del proceso de concesión de un microcrédito en el seno de la institución de microfinanzas, a partir del cual se establecieron las variables explicativas en el mismo. Posteriormente, se analizó el comportamiento que cada variable en cuanto a aumento o reducción de riesgo de impago del cliente evaluado. Condicionada al proceso de concesión del microcrédito, la selección 
de variables finales en este estudio se fijó considerando aquella información susceptible de incrementar o reducir la probabilidad de impago del cliente. Las variables explicativas de partida han sido agrupadas en tres grandes bloques: variables del cliente (cuantitativas y cualitativas), variables de la operación de préstamo y, por último, variables macroeconómicas. La consideración de variables que incorporan la realidad macroeconómica es un aspecto importante en esta investigación, ya que la base de datos inicial de la cartera no las contemplaba, y su incorporación mejoró significativamente los resultados del modelo.

La escasez de datos económico-financieros en los historiales de crédito tuvo que ser completada con información de tipo cualitativo; ello permitió aplicar técnicas estadísticas más flexibles para la incorporación de las variables explicativas. En concreto, la técnica elegida fue la regresión logística binaria, que constituye una herramienta estadística adecuada para el tratamiento de las variables independientes, tanto cuantitativas como cualitativas. Por otro lado, esta técnica ofrece como principal ventaja el cálculo de la probabilidad de incumplimiento del cliente que solicita un crédito, aspecto importante si la entidad de microfinanzas evoluciona de los modelos estándar a modelos basados en calificaciones internas para el cálculo del requerimiento de capital.

La estimación del modelo de credit scoring se realizó mediante el método de introducción por pasos, y aplicando la técnica paramétrica de regresión logística de las variables explicativas sobre la base de las fases y estadios obtenidos en el proceso de concesión de un microcrédito. De esta forma, la investigación realizada diseña un modelo de calificación estadística capaz de predecir correctamente en $78.3 \%$ de los créditos de la cartera de la Edpyme Proempresa, corroborado por un porcentaje similar en el proceso de validación del modelo. A este respecto, las medidas de valoración del modelo globalmente indican un ajuste aceptable en regresión logística.

Finalmente, se comprobó cómo la integración de Basilea II en la gestión del riesgo de crédito en las IMFs supervisadas, mediante métodos IRB frente a los métodos estándar, permite la discriminación de tasas de interés entre clientes. Esto se deriva de las probabilidades de impago, o distinto ratings, que refuerzan la necesidad de diseñar modelos de credit scoring para sus carteras de microcréditos. Incidentalmente, se entiende que el modelo de credit scoring obtenido no va a suponer una limitación de las funciones que realiza el analista de crédito, sino que, por el contrario, debe constituirse en una herramienta complementaria que le permita resumir un conjunto de información cada vez más compleja de analizar.

La presente investigación deja un campo abierto a futuras líneas de investigación sobre el riesgo de crédito en las entidades de microfinanzas. Si la entidad desea emplear el método basado en calificaciones internas (IRB) en su versión avanzada, ella tendrá que hacer uso de sus propias estimaciones internas con objeto de introducir un ajuste en la pérdida en caso de incumplimiento (LGD). Por tanto, una línea abierta al campo de esta investigación consiste en la estimación de la LGD; para ello deberá aplicar una metodología de scoring estadístico. Otra línea de estudio llevaría a analizar el impacto de las técnicas de mitigación del riesgo de impago para las entidades de microfinanzas, previstas en el Acuerdo de Basilea II. 


\section{Referencias}

Allen, L., DeLong, G., \& Saunders, A. (2004). Issues in the Credit Risk Modeling of Retail Markets. Journal of Banking and Finance, 28, 727-752.

Altman, E. I. (1968). Financial Ratios, Discriminant Analysis and the Prediction of Corporate Bankruptcy. Journal of Finance, 23(4), 589-609.

Alvarado, G. (2001). Programas de microcrédito rural para pequeños negocios en Piura: Eficiencia y empoderamiento. Proyecto breve abierto de CIES. Lima, Perú: Consorcio de Investigación Económica y Social.

Bellotti, T., \& Crook, J. (2007). Credit Scoring with Macroeconomic Variables Using Survival Analysis. Credit Research Centre-Management School and Economics. Edinburgh, UK: University of Edinburgh.

Basel Committee on Banking Supervision. (2001). The Internal Ratings-Based Approach (Consultative Document). Basilea, Suiza: Bank for International Settlements-BIS.

Basel Comittee on Banking Supervision. (2004). International Convergence of Capital Measurement and Capital Standards (Consultative Document). Basilea, Suiza: Bank for International Settlements-BIS

Boyle, M., Crook, J. N., Hamilton, R., \& Thomas, L. C. (1992). Methods for Credit Scoring Applied to Slow Payers. In Thomas, L. C., Crook, J. N., Edelman, D. B. (Eds.), Credit Scoring and Credit Control (pp. 75-90). Oxford, UK: Clarendon.

Breiman, L., Friedman, J. H., Olshen, R. A., \& Stone, C. J. (1984). Classification and Regression Trees. Monterey, CA: Wadsworth, Inc.

Carter, C., \& Carlett, J. (1987). Assessing Credit Card Applications Using Machine Learning. IEEE Expert, 2(3), 71-79.

Coffman, J. Y. (1986). The Proper Role of Tree Analysis in Forecasting the Risk Behaviour of Borrowers. Management Decision Systems, MDS Reports 3, 4, $7 \& 9$.
Davis, R. H., Edelman, D. B., \& Gammerman, A. J. (1992). Machine-Learning Algorithms for Credit-Card Applications. Journal of Management Mathematics, 4(1), 43-51.

Dennis, W. (1995). Fair Lending and Credit Scoring. Mortgage Banking, 56(2), 55-59.

Diallo, B. (2006). Un modele de 'credit scoring' pour une institution de microfinance Africaine: le cas de Nyesigiso au Mali. Recuperado de <http:// hal.archives-ouvertes.fr/docs/00/06/91/63/PDF/ s16_05_06diallo.pdf>

Dimitras, A., Zanakis, S., \& Zopouninis, C. (1996). A Survey of Business Failures with an Emphasis on Prediction Methods and Industrial Applications. European Journal of Operational Research, 90, 487-513.

Fisher R. A. (1936). The Use of Multiple Measurements in Taxonomic Problems. Annals of Eugenics, 7(2), 179-188.

Greene, W. H. (1992). A Statistical Model for Credit Scoring (Working Papers 92-29). New York: Leonard N. Stern School of Business, New York University.

Hand, D. J. (1981). Discrimination and Classification. Chichester, UK: Wiley.

Hand, D. J., \& Henley, W. E. (1997). Statistical Classification Methods in Costumer Credit Scoring: A review. Journal of the Royal Statistical Association, 160(A/ Part3), 523-541.

Kim, J. (2005). A Credit Risk Model for Agricultural Loan Portfolios under the New Basel Capital Accord. Dissertation submitted to the Office of Graduate Studies of Texas A \& M University.

Kleimeier, S., \& Dinh, T. (2007). A Credit Scoring Model for Vietnam's Retail Banking Market. International Review of Financial Analysis, 16(5), 471-495.

Kolesar, P., \& Showers, J. L. (1985). A Robust Credit Screening Model Using Categorical Data. Management Science, 31, 123-133. 
Kulkosky, E. (1996). Credit Scoring Could Have a Downside, Experts Say. American Banker, 161(208), 8.

Makowski, P. (1985). Credit Scoring Branches Out: Decision Tree-Recent Technology. Credit World, 75, 30-37.

Mester, L. (1997). What's the Point of Credit Scoring? Business Review (Federal Reserve Bank of Philadelphia), September/October, 3-16.

Milena, E., Miller, M., \& Simbaqueba, L. (2005). The Case for Information Sharing by Microfinance Institutions: Empirical Evidence of the Value of Credit Bureau-Type Data in the Nicaraguan Microfinance Sector. New York: The World Bank, mimeo.

Miller, M., \& Rojas, D. (2005). Improving Access to Credit for Smes: An Empirical Analysis of the Viability of Pooled Data SME Credit Scoring Models in Brazil, Colombia \& Mexico. New York: The World Bank.

Mures, M. J., García, A., \& Vallejo, M. E. (2005). Aplicación del análisis discriminante y regresión logística en el estudio de la morosidad de las entidades financieras. Comparación de resultados. Pecvnia, 1, 175-199.

Orgler, Y. E. (1970). A Credit Scoring Model for Commercial Loans. Journal of Money, Credit and Banking, 2(4), 435-445.

Orgler, Y. E. (1971). Evaluation of Bank Consumer Loans with Credit Scoring Models. Journal of Bank Research, 2, 31-37.

Reinke, J. (1998). How to Lend Like Mad and Make a Profit: A Micro-Credit Paradigm Versus the StartUp Fund in South Africa. Journal of Development Studies, 34(3), 44-61.

Ripley, B. D. (1994). Neural Networks and Related Methods for Classification. Journal of the Royal Statistical Society, Series B(Methodological), 56(3), 409-456.

Rosenberg, E., \& Gleit, A. (1994). Quantitative Methods in Credit Management: A Survey. Operations Research, 42, 589-613.

Superintendencia de Banca y Seguros del Perú - SBS. Reglamento para el requerimiento de patrimonio efectivo por riesgo de crédito. Resolución $N^{\circ} 14.354$ del 30/10/2009. (2009). Lima, Perú: Autor.
Schreiner, M. (1999). A Scoring Model of the Risk of Costly Arrears at a Microfinance Lender in Bolivia. Microfinance Risk Management and Center for Social Development, Washington University in St. Louis. Recuperado de <http://info.worldbank.org/ etools/docs/library/128753/Scoring\%20Model\%20 Costly\%20Arrears\%20Bolivia.pdf $>$.

Schreiner, M. (2000). Credit Scoring for Microfinance: Can It Work?. Microfinance Risk Management and Center for Social Development, Washington University in St. Louis. Recuperado de $<$ http:// www.microfinance.com/English/Papers/Scoring_ Can_It_Work.pdf $>$.

Schreiner, M. (2002). Scoring: The Next Breakthrough in Microfinance. Occasional Paper $N^{\circ} 7$, Consultative Group to Assist the Poorest, Washington D. C.

Sharma, M., \& Zeller, M. (1997). Repayment Performance in Group-Based Credit Programs in Bangladesh: An Empirical Analysis. World Development, 25(10), 1731-1742.

Showers, S. R., \& Chakrin, L. M. (1981). Reducing Uncollectable Revenue from Residential Telephone Customers. Interfaces, 11, 21-31.

Trucharte, C., \& Marcelo, A. (2001). Modelos factoriales de riesgo de crédito: El modelo de Basilea II y sus implicaciones. Actualidad Financiera, 1, 205-218.

Viganò, L. (1993). A Credit-Scoring Model for Development Banks: An African Case Study. Savings and Development, 17(4), 441-482.

Vogelgesang, U. (2003). Microfinance in Times of Crisis: The Effects of Competition, Rising Indebtness, and Economic Crisis on Repayment Behaviour. World Development, 31(12), 2085-2114.

Wiginton, J. C. (1980). A Note on the Comparison of Logit and Discriminant Models of Consumer Credit Behavior. Journal of Financial and Quantitative Analysis, 15(3), 757-770.

Zeller, M. (1998). Determinants of Repayment Performance in Credit Groups: The Role of Program Design, Intra-Group Risk Pooling, and Social Cohesion. Economic Development and Cultural Change, 46(3), 599-620. 


\section{ANEXO A}

\section{Resultados estadísticos modelo Credit Scoring Edpyme Proempresa}

Tabla A1.

Variables de la ecuación en el modelo final

\begin{tabular}{|c|c|c|c|c|c|c|c|c|c|}
\hline & & \multirow{2}{*}{ B } & \multirow{2}{*}{ E.T. } & \multirow{2}{*}{ Wald } & \multirow{2}{*}{$\mathrm{gl}$} & \multirow{2}{*}{ Sig. } & \multirow{2}{*}{$\operatorname{Exp}(B)$} & \multicolumn{2}{|c|}{ I.C. $95,0 \%$ para $\operatorname{EXP}(B)$} \\
\hline & & & & & & & & Inferior & Superior \\
\hline \multirow{10}{*}{$\begin{array}{c}\text { Paso } \\
1^{\mathrm{a}}\end{array}$} & ZONA(1) & -4.98 & 0.081 & 37.967 & 1 & 0.000 & 0.608 & & \\
\hline & SIT_LAB(1) & -1.693 & 0.563 & 9.032 & 1 & 0.003 & 0.184 & 0.519 & $\begin{array}{l}0.712 \\
0.555\end{array}$ \\
\hline & $\mathrm{R} 3$ & -3.062 & 0.552 & 30.782 & 1 & 0.000 & 0.047 & 0.061 & 0.555 \\
\hline & R5 & 1.274 & 0.275 & 21.399 & 1 & 0.000 & 3.576 & 0.016 & 0.138 \\
\hline & CRED_CON2 & -0.070 & 0.017 & 16.244 & 1 & 0.000 & 0.932 & 2.084 & 6.136 \\
\hline & DEST_CRED(1) & -1.088 & 0.136 & 64.501 & 1 & 0.000 & 0.337 & 0.901 & 0.965 \\
\hline & GARANT(1) & -1.064 & 0.083 & 166.253 & 1 & 0.000 & 0.345 & 0.258 & 0.439 \\
\hline & VTOCRED SBS(1) & -4.356 & 0.233 & 348.203 & 1 & 0.000 & 0.013 & 0.294 & 0.406 \\
\hline & $\mathrm{TC}$ & 0.241 & 0.010 & 532.525 & 1 & 0.000 & 1.273 & 0.008 & 0.020 \\
\hline & Constante & 8.703 & 0.639 & 185.375 & 1 & 0.000 & 6022.226 & 1.247 & 1.299 \\
\hline
\end{tabular}

a. Variable(s) introducida(s) en el paso 1: ZONA, SIT_LAB, R3, R5, CRED_CON2, DEST_CRED, GARANT, TOCRED_SBS, TC.

Tabla A2.

Significatividad global del modelo de credit scoring

Prueba ómnibus sobre los coeficientes del modelo

\begin{tabular}{rrccc}
\hline & Chi-cuadrado & gl & Sig. \\
\hline Paso 1 Paso & 1848.966 & 9 & 0.000 \\
Bloque & 1848.966 & 9 & 0.000 \\
Modelo & 1848.966 & 9 & 0.000 \\
\hline
\end{tabular}

Tabla A3.

Bondad del ajuste. Pseudo- $R^{2}$

Resumen de los modelos

\begin{tabular}{cccc}
\hline Paso & $\begin{array}{c}-2 \text { log de la } \\
\text { verosimilitud }\end{array}$ & $\begin{array}{c}\text { Rcuadrado } \\
\text { de Cox y Snell }\end{array}$ & $\begin{array}{c}\text { R cuadrado de } \\
\text { Nagelkerke }\end{array}$ \\
\hline 1 & $3817.438^{\text {a }}$ & 0.364 & 0.485 \\
\hline \multicolumn{3}{c}{$\begin{array}{c}\text { a. La estimación ha finalizado en el número de interación 7 porque las } \\
\text { estimaciones de los parámetros han cambiado en menos de } 0.001 .\end{array}$}
\end{tabular}


Tabla A4

Tabla de contingencias y prueba de Hosmer-Lemeshow

\begin{tabular}{ccccccc}
\hline & & \multicolumn{2}{c}{$\mathrm{Y}=, 00$} & \multicolumn{2}{c}{$\mathrm{Y}=1,00$} & \multirow{2}{*}{ Total } \\
\cline { 2 - 5 } & & Observado & Esperado & Observado & Esperado & \\
\hline \multirow{2}{*}{ Paso } & 1 & 364 & 377.545 & 46 & 31.455 & 409 \\
1 & 2 & 357 & 344.397 & 52 & 64.603 & 409 \\
& 3 & 311 & 315.129 & 98 & 93.871 & 409 \\
& 4 & 272 & 280.427 & 137 & 128.573 & 409 \\
& 5 & 257 & 242.332 & 152 & 166.668 & 409 \\
6 & 229 & 199.638 & 180 & 209.362 & 409 \\
& 7 & 170 & 143.840 & 239 & 265.160 & 409 \\
& 8 & 32 & 79.121 & 377 & 329.879 & 409 \\
9 & 7 & 27.461 & 402 & 381.539 & 409 \\
10 & 17 & 6.111 & 390 & 400.889 & 407 \\
\hline
\end{tabular}

Prueba de Hosmer y Lemeshow

\begin{tabular}{cccc}
\hline Paso & Chi-cuadrado & gl & Sig. \\
\hline 1 & 99.069 & 8 & 0.000 \\
\hline
\end{tabular}

Tabla A5.

Matriz de correlaciones

\begin{tabular}{|c|c|c|c|c|c|c|c|c|c|c|c|}
\hline & & Constant & ZONA(1) & SIT_LAB(1) & R3 & R5 & $\begin{array}{c}\mathrm{CRED}_{-} \\
\mathrm{CON} 2\end{array}$ & $\begin{array}{c}\text { DEST }_{-} \\
\operatorname{CRED}(1)\end{array}$ & GARANT(1) & $\begin{array}{c}\text { VTOCRED_ } \\
\text { SBS(1) }\end{array}$ & $\mathrm{TC}$ \\
\hline Paso & Constante & 1.000 & -0.076 & -0.886 & -0.151 & -0.039 & -0.041 & -0.204 & -0.125 & -0.397 & 0.181 \\
\hline \multirow[t]{9}{*}{1} & ZONA(1) & -0.076 & 1.000 & -0.032 & 0.072 & 0.032 & -0.059 & 0.026 & -0.037 & 0.085 & -0.059 \\
\hline & SIT_LAB(1) & -0.886 & -0.032 & 1.000 & 0.039 & 0.009 & 0.000 & 0.003 & -0.003 & 0.017 & -0.041 \\
\hline & $\mathrm{R} 3{ }^{-}$ & -0.151 & 0.072 & 0.039 & 1.000 & 0.151 & -0.005 & 0.024 & 0.043 & 0.080 & -0.075 \\
\hline & $\mathrm{R} 5$ & -0.039 & 0.032 & 0.009 & 0.151 & 1.000 & -0.246 & -0.019 & -0.093 & 0.015 & 0.067 \\
\hline & CRED_CON2 & -0.041 & -0.059 & 0.000 & -0.005 & -0.246 & 1.000 & -0.122 & -0.063 & -0.077 & -0.051 \\
\hline & DEST_CRED(1) & -0.204 & 0.026 & 0.003 & 0.024 & -0.019 & -0.122 & 1.000 & 0.107 & 0.038 & -0.021 \\
\hline & GARANT(1) & -0.125 & -0.037 & -0.003 & 0.043 & -0.093 & -0.063 & 0.107 & 1.000 & -0.134 & -.0164 \\
\hline & VTOCRED SBS(1) & -0.397 & 0.085 & 0.017 & 0.080 & 0.015 & -0.077 & 0.038 & 0.134 & 1.000 & -0.174 \\
\hline & $\mathrm{TC}$ & 0.181 & -0.059 & -0.041 & -0.075 & 0.067 & -0.051 & -0.021 & -0.164 & -0.174 & 1.000 \\
\hline
\end{tabular}


Tabla A6

Tabla de clasificación

\begin{tabular}{llcccc} 
& & & \multicolumn{3}{c}{ Pronosticado } \\
\cline { 3 - 5 } & & & \multicolumn{2}{c}{$\mathrm{Y}$} & Porcentaje \\
& & & -00 & 1.00 & correcto \\
\cline { 3 - 5 } Paso 1 & $\mathrm{Y}$ & 0.00 & 1798 & 218 & 89.2 \\
& & 1.00 & 671 & 1401 & 67.6 \\
& \multicolumn{2}{c}{ Porcentaje global } & & & 78.3 \\
\hline
\end{tabular}

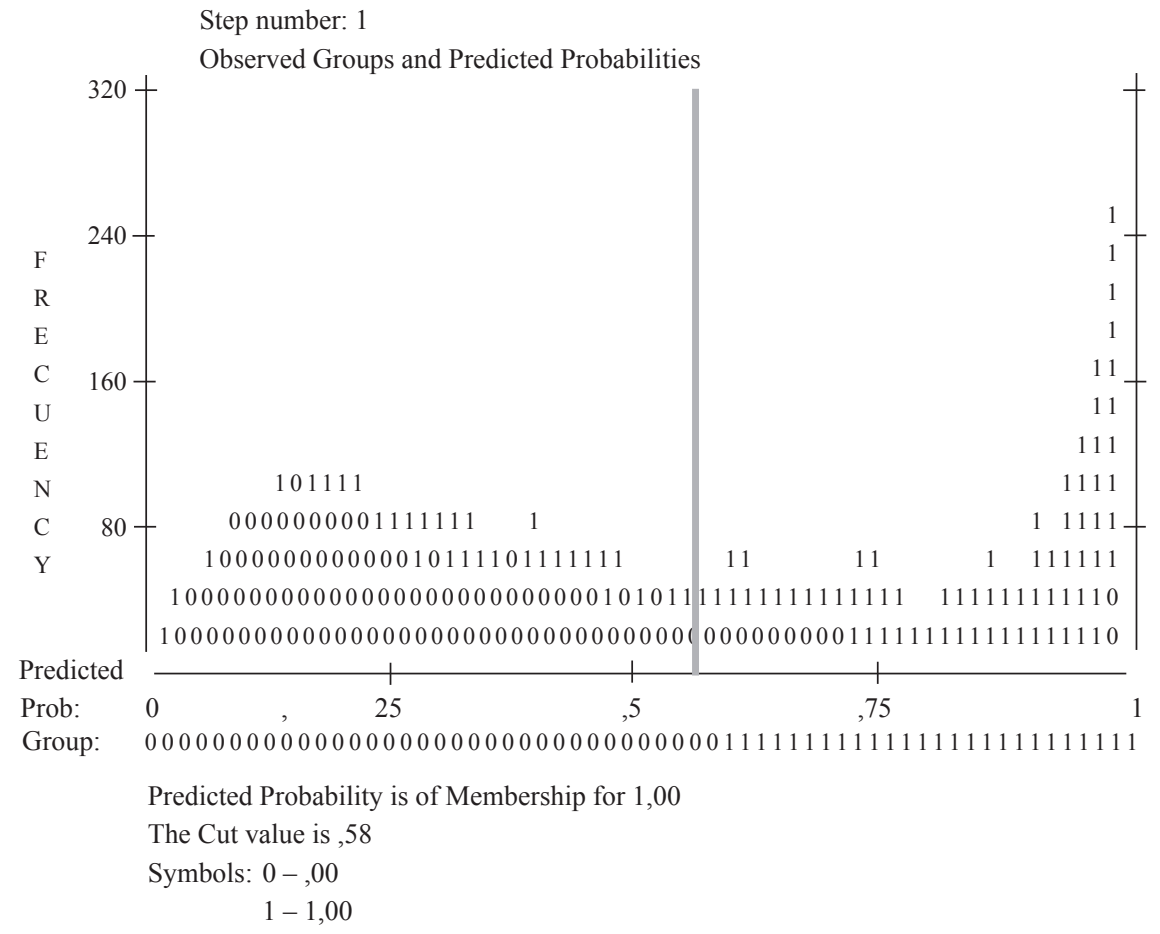

Figura A1. Histograma de probabilidades pronosticadas 


\section{ANEXO B \\ Curva COR}

Tabla B1.

Sensibilidad y especificidad del modelo

\begin{tabular}{cccc}
\hline Punto de corte & Sensibilidad & Especificidad & Total \\
\hline 0.00 & 0.0 & 100.0 & 50.7 \\
0.05 & 3.2 & 98.6 & 51.6 \\
0.10 & 12.7 & 98.1 & 56.0 \\
0.15 & 25.3 & 97.1 & 61.7 \\
0.20 & 37.9 & 94.9 & 66.8 \\
0.25 & 48.0 & 92.3 & 70.5 \\
0.30 & 56.2 & 88.6 & 72.6 \\
0.35 & 63.1 & 84.7 & 74.1 \\
0.40 & 70.2 & 81.1 & 75.7 \\
0.45 & 76.7 & 77.1 & 76.9 \\
0.50 & 82.4 & 73.2 & 77.7 \\
0.55 & 86.7 & 69.7 & 78.1 \\
0.58 & 89.2 & 67.6 & 78.3 \\
0.60 & 90.5 & 66.2 & 78.2 \\
0.65 & 93.3 & 62.5 & 77.7 \\
0.70 & 96.3 & 58.5 & 77.2 \\
0.75 & 97.8 & 54.2 & 75.7 \\
0.80 & 98.8 & 48.2 & 73.2 \\
0.85 & 98.8 & 43.3 & 70.7 \\
0.90 & 98.8 & 36.2 & 67.1 \\
0.95 & 99.1 & 24.3 & 61.2 \\
1.00 & 100.0 & 0.0 & 49.3 \\
\hline
\end{tabular}

Tabla B2.

Área bajo la Curva COR.

Variables resultado de contraste: Probabilidad pronosticada

\begin{tabular}{cccc} 
& & \multicolumn{2}{c}{$\begin{array}{c}\text { Intervalo de confianza } \\
\text { árintótico al } 95 \%\end{array}$} \\
\cline { 3 - 4 } & Error tip. $^{\mathrm{a}}$ & Sig. asintónica & \multicolumn{2}{c}{${ }^{\mathrm{b}}$} & Límite inferior $^{\text {Límite }}$ & $\begin{array}{c}\text { Línerior } \\
\text { super }\end{array}$ & & 0.847 & 0.870
\end{tabular}

a. Bajo el supuesto no paramétrico

b. Hipótesis nula: área verdadera $=1.5$ 


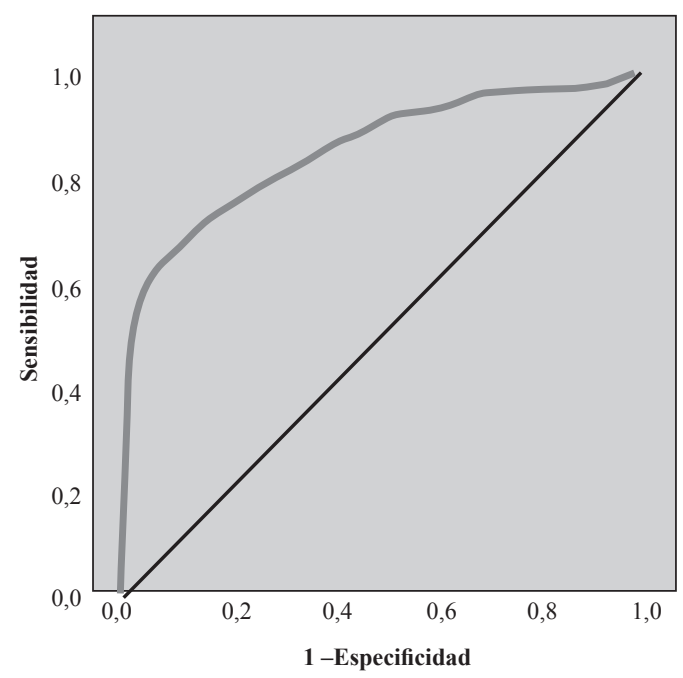

Figura B1. Curva COR 


\section{ANEXO C}

\section{Diagramas de dispersión}

Cambios experimentados por el coeficiente de las variables explicativas
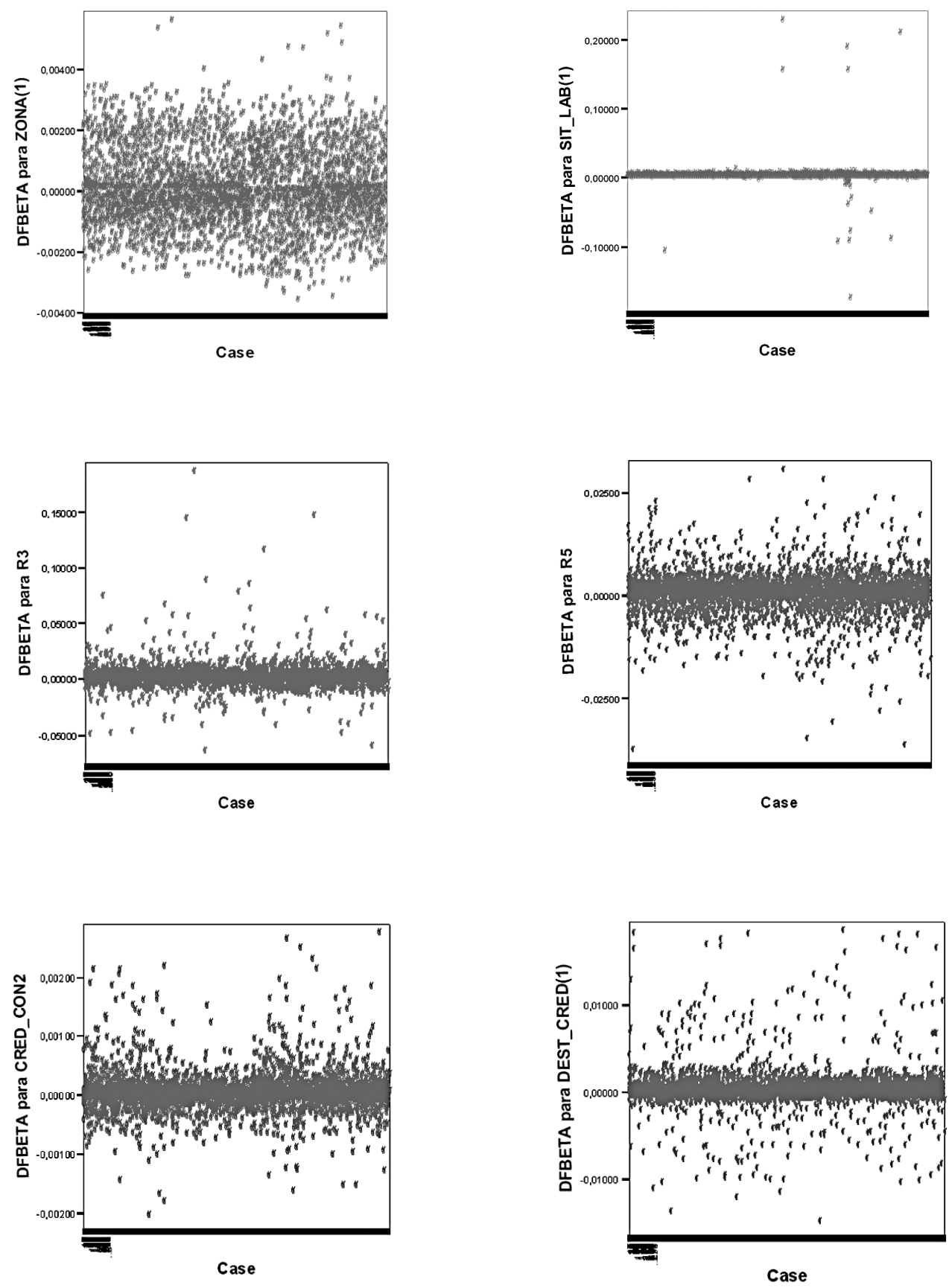

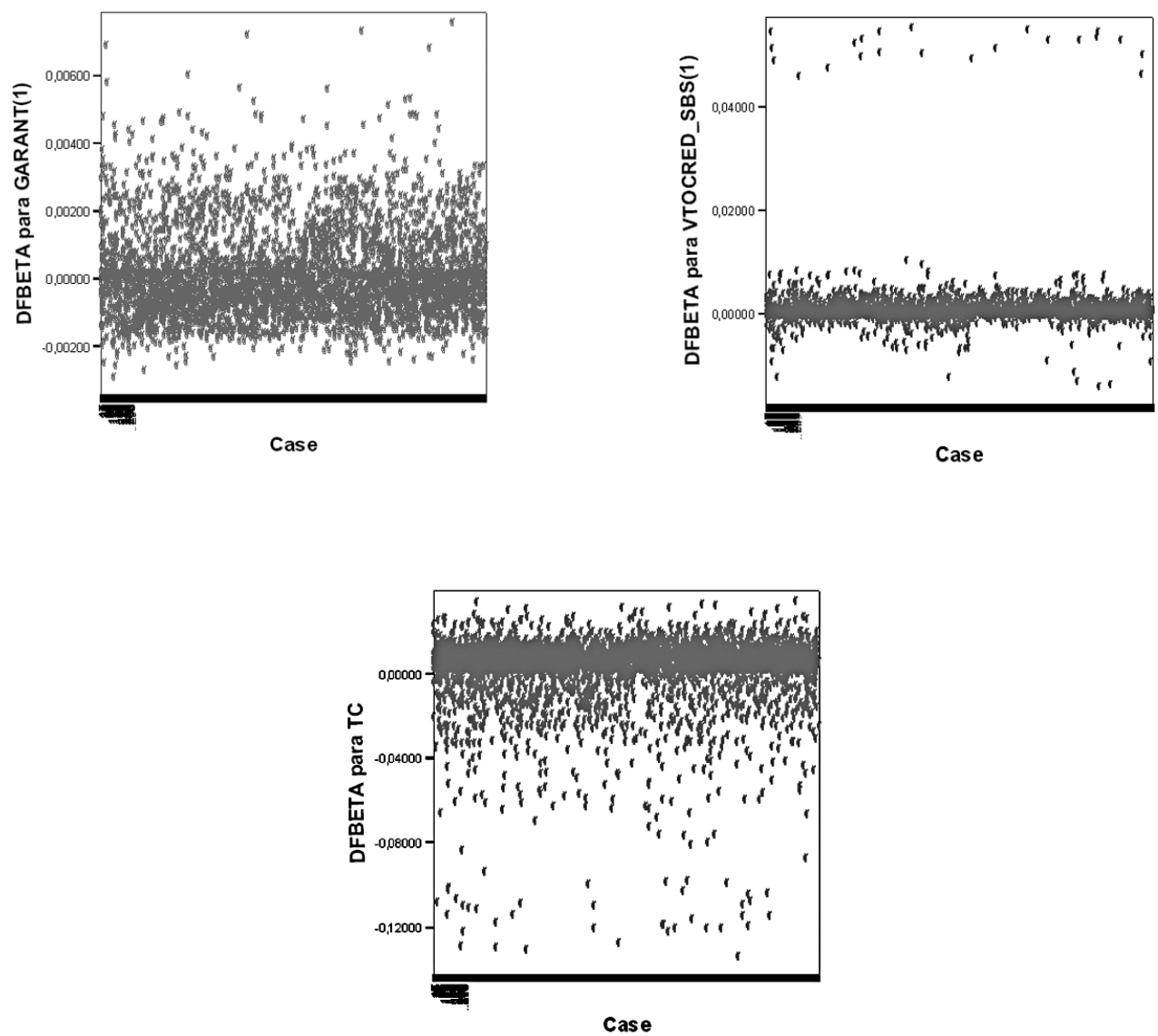